\title{
Die Präferenztheorie in der historischen Phonologie aus junggrammatischer Perspektive
}

\author{
EUGEN HILL
}

\begin{abstract}
The paper deals with the so-called Preference Theory developed in the works of Theo Vennemann and Robert Murray within the scope of historical phonology. The first part of the paper examines the constituting assumptions and claims of the theory. The goal of the preference-based historical phonology - uncovering the motivation for sound changes which the Neogrammarian methodology can merely describe - will be achieved only if the universal preferences are reliably established. It is shown that the procedures which are employed to extract the universal preferences from empirical data do not lead to reliable results. The reason for this is the failure of the Preference Theory to distinguish in a non-arbitrary way between the alleged universally preferred structures and the mere by-products of sound changes with different or unknown motivation. The second part of the paper examines a recently suggested modification of the traditional notion of the exceptionlessness of sound changes. According to Vennemann, the traditional exceptionless sound changes are in fact to be considered as non-exclusive tendencies towards universally more preferred phonological structures. The paper shows that this position is neither based on the core assumptions of the Preference Theory nor supported by the adduced empirical evidence.
\end{abstract}

Keywords: historical phonology, preference laws for syllable structure, sound laws, regularity of sound change, Neogrammarian controversy

\section{Einleitung}

Anfang der 80er Jahre des vergangenen Jahrhunderts hat sich in der historischen Phonologie vor allem der germanischen Sprachen eine neue Forschungsrichtung etabliert. Die Grundidee dieser neuen Richtung besteht in der Annahme, dass Lautwandel, die sich in der Geschichte einer 
jeden Sprache vollziehen, durch Präferenzen für bestimmte Strukturen gesteuert werden. Diese Präferenzen besitzen universellen Charakter, indem sie für alle menschlichen Sprachen gültig sind. Vertreter der Präferenzrichtung erheben in ihren Arbeiten den Anspruch, auf der Grundlage dieser Einsicht erstens dort Erklärungen zu finden, wo die traditionelle, junggrammatische Forschung nur beschreiben kann; zweitens Probleme zu lösen, die mit traditionellen junggrammatischen Methoden nicht gelöst werden konnten. Die dabei aufgestellten Hypothesen können allerdings von Forschern, die der Präferenzrichtung nicht angehören, nicht als Lösungen im eigentlichen Sinne des Wortes akzeptiert werden. Einer der Gründe dafür besteht darin, dass die grundlegenden Annahmen der Präferenztheorie in ihrer gegenwärtigen Form von den Außenstehenden als nicht ausreichend empirisch abgestützt empfunden werden. Ein weiterer Grund ist die theoretische Abgrenzung der Präferenztheoretiker von bestimmten Grundannahmen, die für die traditionelle junggrammatische Forschung die Begriffe „Problem“ und „Lösung“ definieren, wie z. B. die prinzipielle Ausnahmslosigkeit der Lautgesetze. Das Ziel des vorliegenden Aufsatzes besteht darin, die Quellen der Unterschiede zwischen den beiden Richtungen in der historischen Phonologie klarer herauszuarbeiten. Der Aufsatz ist folgendermaßen aufgebaut. In den Abschnitten 1 bis 3 werden die Grundannahmen der Präferenztheorie sowie ihre Vorgehensweise bei der Behandlung des empirischen Materials diskutiert. Es wird sich zeigen, dass die bisherige Präferenztheorie in ihrem zentralen Punkt - der Ermittlung von universellen Präferenzen, die für Lautwandel in einer konkreten Sprache verantwortlich seien - noch keine Erfolge zu verzeichnen hat. Der Abschnitt 4 ist einer Folgerung gewidmet, die aus bestimmten Annahmen der Präferenztheorie gezogen wurde. Die Folgerung betrifft die Diskussion um den ausnahmslosen Charakter der Lautgesetze. Ich werde zeigen, dass diese Folgerung aus den Grundprämissen der Präferenztheorie nicht zwingend folgt und einer kritischen Überprüfung am empirischen Material nicht standhält. Im Abschnitt 5 werden die erzielten Ergebnisse noch einmal zusammengefasst.

\section{Die Grundannahmen der Präferenztheorie}

Die theoretischen Grundlagen des Präferenzzugangs zu den Problemen der historischen Phonologie sind in der Fachliteratur noch nie systematisch dargelegt worden. Die jetzt folgende kurze Übersicht stützt sich in erster Linie auf die Fragmente einer solcher Darstellung in den Arbeiten Murray \& Vennemann (1982), Vennemann (1982, 1983a und b, 1986a und b, 1988, 1993), Murray (1986, 1988, 1991) und Lutz (1988, 1991, 1992). 
Der Präferenzzugang zu Problemen der historischen Phonologie ist in theoretischer Hinsicht auf die verbreitete Prämisse gegründet, dass bestimmte sprachliche Strukturen universell besser oder natürlicher sind als andere. Kriterien zur Ermittlung dessen, welche Strukturen natürlicher sind und welche weniger natürlich, können dabei recht unterschiedlich sein. In der Morphologie - speziell in der Theorie der „morphologischen Natürlichkeit", die bei der Entwicklung der phonologischen Präfe-

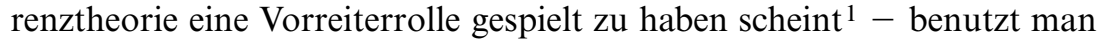
vor allem das Kriterium der Ikonizität. Morphologische Strukturen, deren Bildeweise den strukturellen Eigenschaften der damit kodierten Information besser entspricht, gelten als natürlicher. So gilt es als natürlicher, wenn der Singular eines Nomens weniger Morpheme enthält als der entsprechende Plural. Ferner wird vermutet, dass bessere morphologische Strukturen (a) in den Sprachen der Welt häufiger vorkommen als schlechtere, (b) früher gemeistert werden beim Erstspracherwerb, (c) stabiler sind bei Sprachstörungen toxischer oder pathologischer Natur.

Das Kriterium der Ikonizität ist in der Phonologie aus verständlichen Gründen nicht anwendbar. Die Ermittlung der Qualität oder Natürlichkeit einer phonologischen Struktur muss sich hier auf die anderen drei Kriterien stützen. Die phonologische Struktur A ist somit dann besser oder natürlicher als die phonologische Struktur B, wenn erstens A in den Sprachen der Welt häufiger vorkommt als B; zweitens, wenn Kinder, die ihre Muttersprache erlernen, sich A schneller aneignen als B; drittens, wenn A bei Sprachstörungen verschiedener Natur länger intakt bleibt als B.

Auf dieser Grundlage wurde die präferenzbasierte historische Phonologie entwickelt. Neben der Annahme von universellen Präferenzen liegt ihr noch die folgende These zugrunde: Jeder Wandel in jeder Sprache führt immer von weniger Qualität zu mehr Qualität. Besitzt eine phonologische Struktur A nach einem bestimmten Parameter weniger Qualität als die phonologischen Strukturen B und C, kann sich A von sich aus zu B oder C entwickeln, während die entgegengesetzte unkonditionierte Entwicklung von $\mathrm{B}$ oder $\mathrm{C}$ zu A ausgeschlossen ist. Es wird ferner angenommen, dass in einer Sprache, die alle drei Strukturen A, B und C besitzt, die relativ bessere Struktur B nicht modifiziert wird, bevor die relativ schlechtere Struktur A eine Modifikation erfahren hat. Anders ausgedrückt, die Entwicklung von weniger Qualität zu mehr Qualität setzt bei Strukturen mit der geringsten Qualität an und schreitet graduell $\mathrm{zu}$ immer besseren weiter.

1. Vgl. vor allem Mayerthaler (1981), auf den sich Vennemann (1983a) häufig beruft, ferner Wurzel (1984, 1985) und die Beiträge bei Dressler (1987). 
Dass jeder Lautwandel immer zu mehr Qualität in der betreffenden phonologischen Struktur führt, bedeutet allerdings nicht, dass in den Sprachen der Welt bessere (natürlichere) Strukturen nie durch schlechtere (weniger natürliche) Strukturen ersetzt werden. Im Gegenteil ist eine solche Entwicklung nicht ungewöhnlich. Der Grund dafür liegt in der Tatsache, dass sowohl linear längere Strukturen wie Wort oder Silbe als auch linear kürzere Strukturen, aus denen diese längeren Strukturen sich zusammensetzen, ihre eigenen Präferenzen besitzen. Eine Entwicklung zu mehr Qualität bzw. Natürlichkeit auf der Ebene des Wortes kann als Nebeneffekt zur Verminderung der Qualität oder Natürlichkeit der Silben führen, aus denen dieses Wort besteht. Zum Beispiel führt die Apokope des letzten Vokals in einem Wort der Struktur CVCVCV, also die Entwicklung zu CVCVC, zu einem Wort mit mehr Qualität, weil kürzere Wörter nach einer der Präferenzen besser sind als längere. Zugleich führt diese Entwicklung aber zur Abnahme der Qualität der zweiten Silbe, weil geschlossene Silben nach einer anderen Präferenz schlechter sind als offene. Auch umgekehrt kann mehr Qualität für die Silben zugleich weniger Qualität für das Wort bedeuten, zu dem diese Silben gehören. Zum Beispiel führt die Anaptyxe eines Vokals zwischen die beiden nebeneinander stehenden Konsonanten in einem Wort der Struktur CVCCV, also die Entwicklung zu CVCVCV, zur Zunahme der Qualität der ersten Silbe, die jetzt offen ist, zugleich aber zur Abnahme der Qualität des ganzen Wortes, das jetzt länger ist. Soweit die logische Struktur der Theorie.

Die Präferenztheorie in der historischen Phonologie macht es möglich - die Richtigkeit ihrer Grundannahmen und der ermittelten Präferenzen vorausgesetzt -, die Motivation eines konkreten Lautwandels in einer konkreten Sprache aufzudecken. Die Motivation des Wandels CVCVCV > CVCVC besteht nach der Präferenztheorie in der Zunahme der Qualität auf der Ebene des Wortes. Die Motivation des Wandels CVCCV > CVCVCV besteht in der Zunahme der Qualität auf der Ebene der Silbe. Nachdem die einschlägigen Präferenzen universellen Charakter haben, sind die betreffenden Lautwandel in konkreten Sprachen aus den fundamentalen Eigenschaften der menschlichen Sprache erklärt.

In diesem Punkt muss man einen grundlegenden Unterschied zwischen der Präferenztheorie und der traditionellen junggrammatischen Forschung festhalten. Die junggrammatische Forschung unternimmt bekanntlich keinen Versuch, die Motivation eines bestimmten Lautwandels in einer konkreten Sprache, also den Grund für sein Wirken in dieser Sprache, aufzudecken. Der Aufdeckung der Motivation wohl am nächsten kommt hier die gelegentliche Subsumierung mehrerer unterschiedlicher Lautwandel unter eine größere Tendenz. Man vgl. zum Beispiel die 
Tendenz zur Silbenöffnung im Slavischen, die so verschiedene Lautwandel umfasst wie den Konsonantenschwund im Wortauslaut -VC\# > -V\#, die Auflösungen der Cluster aus Plosiven -VktV- > -VtV-, die Entfaltung der Nasalvokale -VNC- $>$-ęC- oder -oC- und die bekannte Liquidametathese -CVRC- > -CRVC-. Tendenzen dieser Art gelten in der traditionellen Forschung freilich immer nur für eine Sprache, weshalb sich sogleich die Frage nach der Motivation der Tendenzen selbst stellen müsste, wenn man in der Ermittlung der Motivation ein Forschungsziel sähe.

\section{Typologie als Quelle der universellen Präferenzen}

Es ist klar, dass Erklärungen, die die Präferenztheorie für konkrete Lautwandel durch ihre Zuordnung zu universellen Präferenzen liefert, nicht als zuverlässig gelten können, solange die universellen Präferenzen selbst nicht zuverlässig ermittelt sind. Die Ermittlung von Präferenzen scheint aber bisher nicht zu verlässlichen Ergebnissen geführt zu haben. Um das $\mathrm{zu}$ zeigen, soll im weiteren zunächst exemplarisch auf die beiden bereits erwähnten fundamentalen Präferenzen eingegangen werden, die bereits in den grundlegenden Ausführungen Vennemanns zur logischen Struktur der Präferenztheorie eingeführt wurden (1983a: 278-279, vgl. 1986a: 32-34).

Quellen, die über die Präferenzen Aufschluss geben sollten, sind, wie oben festgehalten, erstens die typologischen Daten, zweitens das Material des Erstspracherwerbs, drittens verschiedenartige Sprachstörungen. Der Erstspracherwerb und die Sprachstörungen haben allerdings bei der Ermittlung der universellen Präferenzen in den Schriften der Präferenztheoretiker bisher kaum eine Rolle gespielt, so dass sich eine eingehende Diskussion erübrigt. Auf die Präferenz für leichteren Silbenauslaut wird in erster Linie aufgrund der Tatsache geschlossen, dass Sprachen der Welt von allen möglichen Strukturen der Silbe CV, CVC, CVCC, CVCCC, CVCCCC etc. Silben mit leichterem Auslaut zu bevorzugen scheinen. Dies zeigt sich vor allem darin, dass in einer Sprache, die eine bestimmte Silbenstruktur zulässt, z. B. CVCC, immer auch die Silbenstrukturen mit leichterem Auslaut, also CVC und CV, zugelassen sind. Diese bekannte Tatsache allein reicht allerdings zur Aufstellung einer universellen Präferenz nicht aus. Es ist wiederholt darauf hingewiesen worden, dass es sich bei typologisch verbreiteten, wiederkehrenden Eigenschaften synchroner phonologischer Systeme auch um sekundäre, emergente Erscheinungen handeln kann, die aus dem Wirken typologisch verbreiteter aber anderweitig motivierter Lautwandel resultieren (vgl. Anderson 1981, Blevins \& Garrett 1998, Dolbey \& Hansson 1999, 
heute vor allem Blevins 2004: 278-297, 2005, 2006, 2008). ${ }^{2}$ Konkret in Bezug auf den Silbenauslaut ist z. B. bekannt, dass Silben mit leichterem Auslaut in vielen Sprachen durch relativ rezente Lautwandel aus Silben mit schwererem Auslaut entstehen. Dabei vollziehen sich viele Lautwandel, die zur Reduktion des Silbenauslauts führen, bestimmt nicht oder nicht notwendigerweise auf der Ebene der Silbe. Man vgl. zum Beispiel den Schwund des alten $s$ am Ende des Wortes im Slavischen. Dieses $s-$ in den nah verwandten baltischen Sprachen noch direkt zu beobachten - schwindet im Slavischen nur dann, wenn es das letzte Phonem eines Wortes ist, unabhängig von den prosodischen oder sonstigen Charakteristiken der betreffenden Silbe. Vgl. die folgende Auswahl von Flexionsformen der Nomina im ostbaltischen Litauisch im Vergleich mit ihren Entsprechungen im Altkirchenslavischen, der ältesten bezeugten slavischen Sprache:

(1)

\begin{tabular}{|c|c|c|c|}
\hline Nom. Sg. & $\begin{array}{l}\text { lit. } \\
\text { synŭ }\end{array}$ & $\begin{array}{l}\text { aksl. } \\
\text { sūnùs }\end{array}$ & $\begin{array}{l}\text { Glosse } \\
\text {,Sohn“ }\end{array}$ \\
\hline Gen. Sg. & sūnaũs & synu & \\
\hline Nom. $\mathrm{Sg}$ & naktìs & nošt & ,Nacht ${ }^{6}$ \\
\hline Gen. Sg. & naktiẽs & $n o s ̌ t i$ & \\
\hline Nom. Sg. & piřštas & prǐstŭ & ,Finger \\
\hline Akk. Pl. & pirštùs & prǐsty & \\
\hline Nom. Sg. m. & šìs & $s \breve{l}$ & ,dieser ${ }^{6}$ \\
\hline Akk. Pl. m. & šiuõs & si & \\
\hline
\end{tabular}

Man sieht, dass das letzte Phonem der litauischen Wörter, hier immer das $s$, im Altkirchenslavischen systematisch fehlt, wobei die Akzentverhältnisse oder sonstige prosodische Eigenschaften des Wortes oder seiner letzten Silbe keine Rolle spielen. Da geschlossene Silben auf $s$ in der Mitte des Wortes im Slavischen keine Modifikation erfahren (vgl. Lexeme wie aksl. věsna ,Frühling', maslo ,Öl', město ,Ort' etc.), ist der Schwund des $s$ im Wortauslaut auf der Analyseebene der Silbe nicht zu motivieren, obwohl dieser Schwund zu einer drastischen Zunahme der Silben mit leichterem Auslaut führt. Lautwandel dieser Art müssen zur typologischen Dominanz der Silben mit leichterem Auslaut beigetragen haben.

Neben dem gut dokumentierten und typologisch verbreiteten Konsonantenschwund im Wortauslaut ist insbesondere $\mathrm{zu}$ berücksichtigen,

2. Vgl. Hale $(2003: 361=2007: 140)$ : „There is no a priori reason to believe that synchronic phonological systems and diachronic events are constrained by principles which are at all the same. Indeed, there is a very real danger that many constraints proposed on synchronic phonological systems (proposed because there are no known exceptions in the languages we have studied so far) are in fact not synchronic constraints at all". 
dass bestimmte Konsonanten in den Sprachen der Welt generell wenig stabil sind und zum Schwund in allen oder vielen Stellungen neigen. Der Schwund solcher Phoneme führt in der betreffenden Sprache notwendigerweise zur Zunahme der Silben mit leichterem Auslaut und erzeugt neue offene Silben. Dabei gibt es Lautwandel, die relativ stabile Konsonanten in instabile verwandeln. Man vgl. z. B. den Schwund von $h$ in deutsch sah oder verlieh, durch den viele Silben zu einem leichteren Auslaut kommen. Nachdem das $h$ auch in sahen oder verliehen nicht mehr gesprochen wird, wo es nicht im Auslaut, sondern im Anlaut einer Silbe stand, kann der Schwund von $h$ nicht zu den silbenauslautoptimierenden Lautwandeln zählen. Dabei muss sich das im Inlaut meist geschwundene deutsche $h$, im Mittelalter noch in allen Stellungen gesprochen, über urgerm. $* x$ und $* x^{w}$ aus noch älteren Lauten $* k$ und $* k^{w}$ entwickelt haben, die verhältnismäßig stabile Phoneme sind. Vgl. lateinische Entsprechungen germanischer Wörter mit diesen Spiranten:

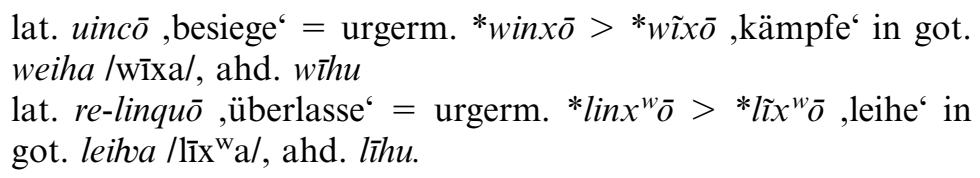

Einen ganz ähnlichen Schwund von $h$ im Inlaut erlebte auch das Griechische, wo dieser Laut allerdings - nach Ausweis des Griechischen selbst und der verwandten Sprachen - auf altes ${ }^{*} s$ zurückgeht. Vgl. die Bildung der $s$-Aoriste zu Verben mit Wurzeln auf Plosive und Resonanten im attischen Dialekt des Altgriechischen:

$$
\begin{aligned}
& \text { griech. } \lambda \dot{\varepsilon} \gamma \omega / \text { légō/ ,sagen' } \rightarrow s \text {-Aorist 1.Sg. urgriech. *é-leg-s-a } \\
& >\text { att. } \stackrel{\varepsilon}{\varepsilon}-\lambda \varepsilon \xi \alpha \text { /éleksa/ } \\
& \text { griech. } \tau \rho \varepsilon \varepsilon \pi \omega / \text { trépō/ ,wenden' } \rightarrow s \text {-Aorist 1.Sg. urgriech. *é- } \\
& \text { trep-s- } a>\text { att. } \ddot{\varepsilon}-\tau \rho \varepsilon \psi \alpha \text { létrepsa/ } \\
& \text { aber } \\
& \text { griech. vé } \mu \omega \text { /némō/ ,zuteilen }{ }^{6} \rightarrow s \text {-Aorist 1.Sg. urgriech. *é-nem- }
\end{aligned}
$$

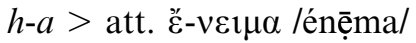

$$
\begin{aligned}
& \text { griech. } \delta \varepsilon \dot{\varepsilon} \rho \omega / \text { dérō/ ,schinden' } \rightarrow s \text {-Aorist 1.Sg. urgriech. *é-der- } \\
& h \text { - } a>\text { att. "̌- } \delta \varepsilon 1 \rho \alpha \text { lédẹra/. }
\end{aligned}
$$

Der Schwund von urgriech. ${ }^{*} h<*_{s}$ im Inlaut zwischen Resonant und

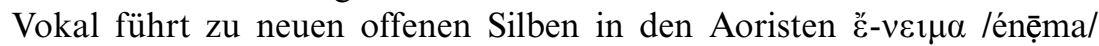
und $\check{\varepsilon}-\delta \varepsilon 1 \rho \alpha$ /édẹra/. Dabei schwindet urgriech. ${ }^{*} h<{ }^{*} s$ auch zwischen Vokalen, wo sein Schwund keine Auswirkungen auf die Beschaffenheit des Silbenauslauts haben kann. Vgl. die Bildung des Plurals und der 
obliquen Kasus zu Stämmen auf $-s$ im homerischen und attischen Altgriechisch:

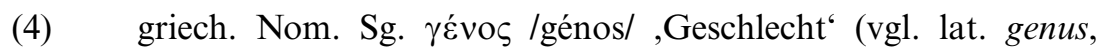
Nom. Pl. genera $<*$ genes- $a$ )

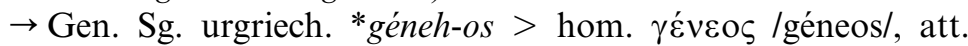
$\gamma$ ćvous /génọ̄s/ (Kontraktion)

$\rightarrow$ Nom. Pl. urgriech. *géneh-a > hom. $\gamma \varepsilon \dot{v \varepsilon \varepsilon \alpha / g e ́ n e a /, ~ a t t . ~ \gamma \varepsilon ́ v \eta ~}$ /génē/ (Kontraktion).

Sekundäre offene Silben entstehen durch den Schwund eines inlautenden $h$ auch im Ketischen, einer jenissejischen Sprache in Südsibirien. Hier entwickelt sich das $h-$ in den Aufzeichnungen aus dem 18. Jh. noch in allen Stellungen belegt - aus einem (wahrscheinlich aspirierten) ${ }^{*} p$. Verwandte Sprachen weisen in ihren Entsprechungen der ketischen Lexeme und Morpheme mit $h$ Labiale auf: man findet $f$ im Jugischen, $f$ oder $p^{h}$ im Kottischen und Arinischen, $p$ oder $p f$ im Pumpokolischen (ausführlich Hill i.Dr. mit Lit.). Vgl. z. B. ket. Karitiv Sg. húnàn < *húnhàn ,ohne Tochter' mit einer offenen Silbe neben jug. fún-fàn und kott. fun-fun, die aus zwei geschlossenen Silben bestehen. Ket. $h$ schwindet auch zwischen Vokalen, wo sein Ausfall keine Auswirkungen auf die Silbenstruktur hat. Man vgl.

ket. (18. Jh.) kóhil $>$ ket. (20. Jh.) kû:l = jug. kúfỳr ,glühende Kohlen ${ }^{\circ}$

ket. (18. Jh.) éhel $>$ ket. (20. Jh.) ê:l = jug. éfỳr ,Preiselbeere

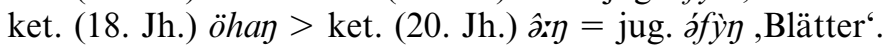

Die angeführten drei Fälle zeigen, dass stabile Konsonantenphoneme wie $k, s$ oder $p$ sich in natürlichen Sprachen in das weit weniger stabile $h$ entwickeln können, dessen Schwund in einem weiteren Schritt automatisch neue offene Silben generiert.

Man sieht also, dass in verschiedenen Sprachen offene Silben sekundär entstehen können durch Lautwandel, die primär nichts mit der Struktur der Silbe zu tun haben. Lässt man das typologische Kriterium bei der Ermittlung der Präferenzen gelten, muss man somit alle diejenigen Sprachen aus der Ermittlung der Präferenzen für die Struktur der Silbe ausschließen, die Lautwandel besagten Typs erlebt haben, außerdem auch alle diejenigen, deren Geschichte nicht ausreichend bekannt ist. Sonst würde die Motivation für einen konkreten Lautwandel mit $\mathrm{CVC}>\mathrm{CV}$ Effekt in einer konkreten Sprache letztendlich in der trivialen Aussage bestehen, dass solche Lautwandel - aus welchem Grund auch immer in typologischer Hinsicht unauffällig sind. Nun ist eine Untersuchung 
von Sprachen mit bekannter Geschichte aber ohne rezente Lautwandel des zu erklärenden Typs niemals durchgeführt worden. Die Präferenz für offene Silben ist somit empirisch nicht ausreichend abgesichert. ${ }^{3}$

Wenn man versucht, die Präferenz für offene Silben bzw. leichteren Silbenauslaut durch konkretes sprachliches Material zu belegen, wird die Tragweite des Problems sofort deutlich. Von allen mir bekannten Sprachen mit gut erforschter Geschichte hat das Litauische wohl am wenigsten Lautwandel durchgemacht, die den Auslaut der Silbe verändern. Wenn man die Lautwandel berücksichtigt, die in der jüngsten Vorgeschichte des Litauischen den Auslaut von Silben veränderten, stellt man fest, dass die wichtigeren davon nichts mit der Silbenstruktur zu tun haben. Es handelt sich dabei um verschiedene Arten von Konsonantenschwund:

- Schwund der sogenannten „Laryngale“ (wahrscheinlich pharyngale Spiranten) vor und nach anderen Konsonanten (vorbalt. *uiHrós > urbalt. *uīras > lit. výras ,Mann'); die „Laryngale“ schwinden auch zwischen Vokalen und im Wortanlaut;

- Schwund der Plosive im Wortauslaut (vorbalt. Abl. Sg. *-ād\# > urbalt. Gen. Sg. *-ä\#> lit. -o\#); im Wortinlaut bleiben die Plosive allerdings immer erhalten, so dass auch dieser Lautwandel in keinem ursächlichen Zusammenhang mit der Silbenstruktur stehen kann;

- Schwund der Nasale vor Nichtplosiven und im Wortauslaut (Akk. Sg. vorbalt. ${ }^{*}$-om\# > urbalt. *-an\# > lit. -a\#); Nasale bleiben im Inlaut vor Plosiven immer erhalten, so dass auch dieser Lautwandel nicht einfach durch eine Präferenz für leichteren Silbenauslaut hervorgerufen sein kann.

Diese Lautwandel erzeugten aus (C)CVCC-Silben sekundäre (C)CVCSilben und aus (C)CVC-Silben sekundäre (C)CV-Silben, ohne sich notwendigerweise auf der Ebene der Silbe zu vollziehen. Andererseits führt die Auflösung der alten silbischen Resonanten in Abfolgen des Typs Vokal plus Resonant - vorbalt. *mntís > lit. mintìs ,Gedanke“ oder vorbalt. *pŕstos $>$ lit. piřstas ,Finger ${ }^{*}$ - zu sekundären (C)CVC- und sekundären (C)CVCC-Silben.

Es ist klar, dass ein Großteil der litauischen Silben mit leichterem Auslaut, wie ein Teil der Silben mit schwererem Auslaut, auf das Wirken

3. Das Ausschließen von Vorgängen, die zur Diskussion stehende Phänomene beeinflussen, ohne notwendigerweise durch diese Phänomene hervorgerufen zu sein, gehört zur Standardpraxis der wissenschaftlichen Forschung. So werden z. B. die Bewegungsgesetzte anhand von Gegenständen untersucht, die keinen eigenen Antrieb haben. Es ist zulässig, bei einem Pendelexperiment eine Billardkugel zu benutzen, nicht aber einen lebenden Maikäfer. 
solcher Lautwandel zurückgehen, die eine Zuordnung zu silbenstrukturoptimierenden Strategien nicht zulassen. Wenn in der Vorgeschichte des Litauischen keine (C)CV-Silben möglich gewesen wären, hätten sie sich jetzt infolge solcher Lautwandel im Sprachsystem fest etabliert. Sekundäre offene Silben findet man heute vielfach in besonders frequenten Flexionsausgängen wie dem Nom. Sg. der frequentesten Stammklassen der Feminina, ${ }^{4}$ Akk. und Gen. Sg. der frequentesten Stammklassen der Maskulina, ${ }^{5}$ 3. Person Präsens und Präteritum aller Numeri beim Verb. ${ }^{6}$

Genauso problematisch ist die Aufstellung der zweiten fundamentalen Präferenz, der Präferenz für kürzere Äußerungen, die auf der Analyseebene des Wortes operieren soll. Diese Präferenz spielt bei der Ausarbeitung der Präferenzgesetze für Silbenstruktur in Vennemann (1983b, 1986a und 1988) die entscheidende Rolle, die in den Sprachen der Welt sehr verbreiteten Synkopen und Apokopen zu erklären. Diese Lautwandel zeitigen Ergebnisse, die allen Präferenzen für die Struktur der Silbe zuwiderlaufen. Nun wird die Präferenz für Kürze des Wortes in dieser allgemeinen Form durch typologische Beobachtungen an synchronen sprachlichen Systemen nicht nahe gelegt. Dass eine Sprache, die linear längere Wörter kennt, z. B. solche aus 4 oder 5 Phonemen, unbedingt auch kürzere Wörter aus 3 bis 1 Phonem haben muss, trifft in dieser Form wohl nicht zu. Aus einem einzigen Phonem bestehen z. B. im Deutschen nur die Interjektionen $a h$, äh und $o h$. Bei diesen Interjektionen scheint es sich aber nicht um deutsche Lexeme zu handeln, sondern vielmehr um Elemente nichtverbaler Kommunikation, die fast in jeder europäischen Sprache mit der gegebenen Lautung und Funktion vorhanden sind (vgl. z. B. russisch $a, \dot{e}, o$ oder litauisch $\tilde{a}, \tilde{e}, \tilde{o}$ ).

Eine universelle Präferenz für kürzere Äußerungen lässt sich somit nicht halten, wenn man die Länge sprachlicher Äußerungen in Phonemen misst. Das Aufstellen dieser Präferenz ist aber auch dann problematisch, wenn man der typologischen Betrachtung linear längere Strukturen zugrundelegt, z. B. die Silbe. Es scheint tatsächlich so zu sein, dass in Sprachen mit mehrsilbigen Wörtern immer auch einsilbige Wörter möglich sind, wobei es möglicherweise auch Sprachen gibt, die keine Wörter aus mehr als einer Silbe kennen (so vielleicht das Altchinesische, wenn man von durchsichtigen Komposita absieht). Einsilbige Wörter scheinen somit auf den ersten Blick typologisch bevorzugt. Es ist aber durchaus möglich, dass diese bevorzugte Stellung wieder das Ergebnis von Lautwandeln ist, die sich durch eine Präferenz für weniger Silben

4. Nom. Sg. vorbalt. $*_{-} a h_{2}$ und $*_{-}-i h_{2}>$ urbalt. ${ }^{*}-\bar{a}$ und $*_{-}-\bar{l}>$ lit. $-a$ und $-i$.

5. Akk. Sg. vorbalt. $*_{-o m} *_{-i m} *_{-u m}>$ urbalt. $*_{-} a n, *_{-i n} *_{-}-u n>$ lit. $-a,-i,-u$; vorbalt. Abl. Sg. $*-\bar{a} d>$ urbalt. Gen. Sg. $*-\bar{a}>$ lit. $-o$.

6. Vorbalt. 3. Sg. Injunktiv $*_{-}(\underset{n}{)}) e d>$ urbalt. 3. Präsens $*_{-}(\underset{-}{i}) a>$ lit. $-(i) a$. 
im Wort nicht motivieren lassen. Man vgl. z. B. die bekannte Apokope von urgermanisch *-uz im Nom. Sg. der altsächsischen und althochdeutschen Substantive. Dieses urgerm. * ${ }^{*} u z$ - im nahverwandten Gotischen noch als -us erhalten - wird im Altsächsischen und Althochdeutschen bekanntlich oft apokopiert. Die Apokope findet aber nur nach schwerer Silbe statt, nach leichter Silbe bleibt ${ }^{*}-u z$ als $-u$ eigentlich erhalten:

$\begin{array}{lllll}\text { Urgermanisch } & \text { Gotisch } & \text { Altsächsisch } & \text { Althochdeutsch } & \text { Glosse } \\ \text { *lustuz } & \text { lustus } & \text { lust } & \text { lust } & \text {,Lust } \\ \text { *flōðuz } & \text { flōdus } & \text { flōd } & \text { fluot } & \text {,Flut } \\ \text { *skilduz } & \text { skildus } & \text { skild } & \text { skilt } & \text {,Schild } \\ \text { *sunuz } & \text { sunus } & \text { sunu } & \text { sunu } & \text {,Sohn“ } \\ \text { *siðuz } & \text { sidus } & \text { sidu } & \text { situ } & \text {,Sitte“ } \\ \text { *magus } & \text { magus } & \text { magu } & - & \text {,Junge'. }\end{array}$

Es ist offensichtlich, dass die Apokope von *-uz in Lexemen wie as. lust, flöd und skild nicht einfach durch das Streben nach weniger Silben im Wort motiviert werden kann: Eine solche Apokope hätte die zweite Silbe überall eliminiert, auch in as. sunu, sidu und magu. Der Lautwandel dient vielmehr, wie längst erkannt, der Einbettung der betreffenden Wörter in den altwestgermanischen zweimorigen prosodischen Template, bei dem die Zahl der Silben keine Rolle spielt $\left(\mu \mu=\mu^{\$} \mu\right) .{ }^{7}$ Die Apokope von urgerm. ${ }^{*}-u z$ führt aber dazu, dass in den betreffenden Sprachen als Nebeneffekt neue einsilbige Wortformen entstehen.

Bezieht man nun die angenommene Präferenz für kürzere Äußerungen auf die Zahl der Moren im Wort, stellt man wieder fest, dass der synchrone typologische Befund eine solche Präferenz nicht trägt. Es gibt durchaus Sprachen, in denen nur Wörter aus mehr als einer More möglich sind. So ist der Vokal in allen einsilbigen Lexemen mit vokalischem Auslaut im Avestischen, einer altostiranischen Sprache, obligatorisch lang und die Wörter entsprechend zweimorig (vgl. Hoffmann \& Forssman 2004: 54). Das gleiche gilt für den japanischen Dialekt von Osaka, wenn man von den Klitika absieht, die ja selbständig kein prosodisches Wort bilden können.

Beide phonologischen Präferenzen, die bereits in der grundlegenden Skizze der Präferenztheorie bei Vennemann (1983a) aufgestellt und seitdem mehrfach zur Erklärung der Lautwandel in verschiedenen Sprachen der Welt herangezogen wurden, entbehren somit einer nachprüfbaren

7. Zur Rolle der prosodischen Templates beim Lautwandel vgl. allgemein vor allem Macken \& Salmons (1997), speziell im Westgermanischen jetzt Smith (2007) mit Lit. 
empirischen Grundlage. Bei der Aufstellung dieser Präferenzen wurde nicht ausreichend berücksichtigt, dass typologisch häufige Eigenschaften phonologischer Systeme in vielen Fällen durch rezente Lautwandel zustande kommen, die mit diesen Eigenschaften primär in keinem Zusammenhang stehen.

Die Möglichkeit von emergenten, epiphänomenalen Strukturen in den synchronen Lautsystemen von Sprachen wird auch in der Präferenztheorie durchaus anerkannt: „Es kommt gewiß vor, daß in einer Sprache durch phonologisch oder konzeptuell-analogisch motivierte Veränderungen komplexe Artikulationen, ja sogar disfavorisierte Phonemsysteme entstehen, die dann im Sinne phonetischer Präferenzen abgebaut werden“ (Vennemann 1983a: 279). Solchen emergenten Lautstrukturen kommt in der Präferenztheorie, wie oben bereits festgehalten, sogar die wichtige Rolle zu, zu erklären, warum die Sprachen der Welt durch optimierende Lautwandel nicht bereits den bestmöglichen Zustand erreicht haben. Was fehlt, ist ein verlässliches Mittel, die universell präferierten synchronen Zustände, also das Ziel der speziell darauf gerichteten Lautwandel, von den disfavorisierten synchronen Zuständen zu unterscheiden, denen epiphänomenaler Charakter zugeschrieben wird.

Dieses Problem scheint grundsätzlich allen teleologischen Modellen des Lautwandels inhärent zu sein, die mit universell besseren oder natürlicheren Strukturen bzw. dem Begriff „markiert“ operieren. GleichermaBen betroffen ist z. B. auch die primär synchron orientierte, in letzter Zeit aber häufig zur Analyse der Lautwandel herangezogene Optimalitätstheorie. Kager stellt in seiner systematischen Darstellung dieser Theorie fest, dass ,,any exclusively typology-based definition of universality runs the risk of circularity: certain properties are posited as ,unmarked' simply because they occur in sound systems with greater frequency than other ,marked" properties" (Kager 1999: 11-12). Kager sucht den Ausweg aus dem Dilemma in der Phonetik. Die universellen „markedness constraints" der Optimalitätstheorie sind abzusichern durch Beobachtungen zur phonetischen Komplexität der betreffenden Lautstrukturen, wobei man erwartet, dass weniger komplexe bzw. artikulatorisch weniger aufwendige Strukturen auch weniger markiert sind und aus diesem Grund in den Sprachen der Welt häufiger vorkommen (vgl. ähnlich Hayes 1999, Kirchner 2000). In diesem Punkt trifft sich die Optimalitätstheorie mit der natürlichen Phonologie, deren universelle Präferenzen sich direkt aus Überlegungen zur leichteren Artikulation und/oder Perzeption ableiten (vgl. Donegan \& Stampe 1979, Donegan 1993). Dies führt bekanntlich zu Schwierigkeiten bei der Erklärung derjenigen Lautwandel, die in diesem Sinne nicht optimierend sind (vgl. Blevins 2004: 
71-77, Yu 2004). ${ }^{8}$ Die Präferenztheorie von Vennemann und Murray schlägt einen anderen Weg ein, der im jetzt folgenden Abschnitt kurz dargestellt sei.

\section{Präferenzen aus Lautwandeln}

Die Mehrheit der universellen Präferenzen zur Erklärung konkreter Lautwandel in konkreten Sprachen ist in der präferenztheoretischen historischen Phonologie nicht durch synchrone typologische Betrachtungen, sondern durch ein ganz anderes Verfahren gewonnen. Dieses Verfahren ergibt sich besonders klar aus der Darstellung der Präferenzen für die Struktur der Silbe bei Vennemann (1988) und den Erläuterungen dazu in Vennemann (1993). Hier werden die Präferenzen für die Struktur der Silbe nicht synchron ermittelt, um dann die Lautwandel in konkreten Sprachen zu erklären, sondern man sammelt Lautwandel mit ähnlichem Ergebnis, zum Beispiel Lautwandel, die offene Silben erzeugen. Da man angenommen hat, dass jeder Lautwandel Zunahme der Qualität einer Struktur auf einer bestimmten Analyseebene nach einem bestimmten Parameter bedeutet, wird das Ergebnis dieser Lautwandel als besser eingestuft als ihr Ausgangspunkt. So ergibt sich aus den Wandeln, die offene Silben erzeugen, wie zum Beispiel dem Konsonantenschwund im Wortauslaut, eine Präferenz für offene Silben, die für alle Sprachen der Welt gelten soll. Diese Präferenz erklärt nun nach der Meinung der Präferenztheoretiker die Lautwandel mit dem Effekt der Silbenöffnung in einer konkreten Sprache. ${ }^{9}$

Dieses Vorgehen bei der Ermittlung der universellen Präferenzen kann aus zwei Gründen prinzipiell nicht zu verlässlichen Ergebnissen führen.

Erstens könnte eine universelle Präferenz nur dann aus konkreten Lautwandeln zuverlässig ermittelt werden, wenn jede Änderung in einer phonologischen Struktur eindeutig einer bestimmten Analyseebene zugeordnet und der relevante Parameter aus der Änderung selbst objektiv

8. Diese inhärente Schwäche der universalistischen teleologischen Lautwandelmodelle ist in meinen Augen aber kein Grund, eine Theorie des Lautwandels anzustreben, die frei von jeder Teleologie wäre (vgl. z. B. die ,evolutionäre Phonologie“ von Blevins \& Garrett 1998, Blevins 2004, 2006). Es ist nicht klar, wie im Rahmen eines solches teleologiefreien Systems Tendenzen von der Art der slavischen Tendenz zur Silbenöffnung erklärt werden können.

9. Vennemann (1993: 325): ,[...] since I am convinced that my theory is correct, I will collect as many instances of language change as I can and arrange the states they start from, and those they lead to, on scales which are to reflect the parameter on which I assume the change to have taken place; and, of course, I make the resulting state or property rank higher on the scale of linguistic quality than the starting state or property". 
ersichtlich wäre. In Wirklichkeit ist weder das eine noch das andere der Fall. Es ist nur selten möglich, einen konkreten Lautwandel eindeutig einer bestimmten Analyseebene zuzuordnen. Auf welcher Analyseebene spielt sich zum Beispiel die verbreitete Auflösung der heterosyllabischen Konsonantencluster durch Anaptyxe eines Kurzvokals $\mathrm{CVC}^{\$} \mathrm{CVC}>$ $\mathrm{CV}^{\$} \mathrm{CV}^{\$} \mathrm{CVC}$ ab? Auf der Ebene der ersten Silbe, die dadurch einen leichteren Auslaut bekommt, oder auf der Ebene des Silbenkontakts, der sich dadurch nicht unerheblich ändert? Oder vielleicht auf der Ebene des Wortes, das eine andere Silbenstruktur bekommt? Eine objektive Entscheidung scheint hier nicht möglich.

Ist die Zuordnung zu einer Analyseebene weniger problematisch, stellt sich sogleich die Frage nach dem einschlägigen Parameter. Es ist zum Beispiel klar, dass die Synkope des letzten Vokals in einem Wort der Struktur $\mathrm{CV}^{\$} \mathrm{CVC}$, also der Wandel $\mathrm{CV}^{\$} \mathrm{CVC}>\mathrm{CVCC}$, auf der Ebene der betreffenden Silbe nicht zu motivieren ist. Eine solche Motivation könnte wohl nur in der Sentenz bestehen, dass Null präferenztheoretisch besser ist als Silbe, was wohl nicht ernsthaft angenommen werden kann. Es bleibt die Ebene des Wortes. Wie ermittelt man aber den relevanten Parameter, nach dem die Qualität des Wortes zunimmt? Handelt es sich um die Länge des Wortes in Silben oder die Länge des Wortes in Moren? Oder vielleicht um die Länge des Wortes in Phonemen? Oder um die Beschaffenheit des Wortauslauts? Von dieser Entscheidung hängt ab, ob es sich bei ähnlichen Änderungen $\mathrm{CV}^{\$} \mathrm{CV}>\mathrm{CVC}$ und $\mathrm{CV}^{\$} \mathrm{CVR}>$ $\mathrm{CV}^{\$} \mathrm{CR}$ (mit silbischem Resonanten im Auslaut) um denselben Lautwandel handelt oder nicht.

Zweitens ist die Ermittlung der universellen Präferenzen aus konkreten Lautwandeln, die durch diese Präferenzen zugleich zu erklären sind, ein zirkelschlussverdächtiges Verfahren. Sie wäre es nicht, wenn die Grundprämisse, nämlich der optimierende Charakter eines jeden Lautwandels, entweder selbstverständlich wäre oder argumentativ wahrscheinlich gemacht werden könnte. Selbstverständlich ist diese Annahme mit Sicherheit nicht: Erst vor relativ kurzer Zeit gingen viele Sprachforscher davon aus, dass Sprachen grundsätzlich eher degenerieren. Argumente zugunsten des grundsätzlich optimierenden Charakters jedes Lautwandels sind nicht möglich, solange nicht unabhängig ermittelt ist, welche Strukturen universell besser und welche universell schlechter sind.

Die Zirkularität der Ermittlung von Präferenzen aus den Lautwandeln, die durch diese Präferenzen zu erklären sind, wird auch von Vennemann (1993: 325-330) konstatiert. Nach Vennemann kann das Verfahren allerdings doch nicht als zirkulär gelten, und zwar aus den folgenden drei Gründen.

- Erstens komme man bei der Untersuchung der Lautwandel, die in einer Reihe von Sprachen eine bestimmte Struktur nach einem be- 
stimmten Parameter verändern, schnell zu dem Schluss, dass alle Wandel dieser Art in dieselbe Richtung gehen.

Dieses Argument würde einleuchten, wenn die Änderungen in phonologischen Strukturen immer eindeutig einer bestimmten Analyseebene zugeordnet wären und die Ermittlung der Parameter irgendwie objektiv erfolgte. Solange das nicht der Fall ist, besteht immer die Möglichkeit, unter die Entwicklung einer Lautstruktur auf einer Analyseebene nach einem Parameter nur diejenigen Lautwandel zu subsumieren, die in eine bestimmte Richtung gehen. Bei allen anderen kann man entweder die betreffende Struktur einer anderen Analyseebene zuweisen oder die Entwicklung als Änderung nach einem anderen Parameter interpretieren. ${ }^{10}$

Beide Verfahren - Änderung der Beschreibungsebene und Einführung neuer Parameter - werden in der praktizierten Präferenztheorie tatsächlich angewendet, um die störenden Ausnahmen zu beseitigen. Die häufige Entwicklung des heterosyllabischen Clusters /ld/ zu /1l/ zwischen Vokalen verstößt zum Beispiel gegen die Präferenz für den Anlaut der zweiten Silbe, nach der mehr konsonantische Stärke ${ }^{11}$ mehr Qualität bedeutet, zugleich aber auch gegen die Präferenz des Silbenkontakts, nach der mehr Unterschied in der konsonantischen Stärke besser ist als weniger Unterschied. Um Fällen dieser Art beizukommen, führt Vennemann (1986a: 44-54, 1988: 35-40) im Anschluss an Murray (1982) ein Präferenzgesetz für Angleichung der konsonantischen Stärke ein, das besagt, dass der Verlust der Stärke durch den stärkeren Konsonanten zu mehr Qualität der Assimilation führt. Dadurch wird sowohl eine neue Beschreibungsebene eingeführt, auf der sich der Lautwandel vollzieht, nämlich die Ebene der Assimilation, als auch ein neuer Parameter, nämlich die Richtung, in die sich die konsonantische Stärke des stärkeren Elements eines Clusters bei Assimilationen entwickelt. Wendet man dieses Verfahren bei der Klassifizierung der Lautwandel konsequent an, kann es Gegenevidenz prinzipiell nicht geben.

Dies soll am Beispiel der Entwicklung des heterosyllabischen Resonantenclusters / $\mathrm{rl} /$ nach dem Parameter der universellen konsonantischen Stärke veranschaulicht werden. Heterosyllabisches /rl/ wird zu /rr/ in Dialekten des Italienischen, aber zu /11/ in Dialekten des Altnordischen. Es

10. Dies übersieht z. B. Auer (2001), der gegen die universelle Präferenz für leichteren Silbenauslaut Strukturen ins Feld führt, die durch Synkopen entstanden sind. Auer schlägt vor, solche Strukturen auf der Analyseebene des Wortes zu betrachten, was der Position Vennemanns sehr nahe kommt. Der Unterschied besteht darin, dass die Analyseebene Silbe oder Wort - bei Auer durch den allgemeinen prosodischen Typ der betreffenden Sprache festgelegt ist: Er unterscheidet zwischen Silbensprachen und Wortsprachen.

11. Zum Begriff ausführlich Vennemann (1982), Murray \& Vennemann (1982 und 1983) und Vennemann (1986a: 34-36). 
ist klar, dass sich der Cluster /rl/ in den betreffenden Sprachsystemen in entgegen gesetzte Richtungen entwickelt. Da $/ \mathrm{r} /$ in der Präferenztheorie schwächer ist als $/ 1 /{ }^{12}$ wird der Unterschied in der konsonantischen Stärke in den beiden Fällen abgebaut, was gegen die Präferenz für den Silbenkontakt verstößt. Die Entwicklung von $/ \mathrm{rl} / \mathrm{zu} / \mathrm{rr} /$ verstößt dabei auch gegen die Präferenz für den Anlaut der zweiten Silbe. Diese Entwicklung führt aber zu mehr Qualität bezüglich der Assimilation der konsonantischen Stärke in einem Cluster. Die Entwicklung der konsonantischen Stärke bei Assimilationen in einem Cluster ist somit der für den Wandel $/ \mathrm{rl} />/ \mathrm{rr} /$ relevante Parameter. Die Entwicklung von $/ \mathrm{rl} /$ $\mathrm{zu} / 11 /$ verstößt aber auch gegen die Präferenz für die Entwicklung der konsonantischen Stärke bei Assimilationen in einem Cluster, weil nach dieser Präferenz Abnahme der konsonantischen Stärke beim stärkeren Element besser ist als Zunahme beim schwächeren. In dieser - eigentlich auswegslosen - Situation steht es der Präferenztheorie aber frei, den Lautwandel $/ \mathrm{rl} / />/ 11 /$ auf einer anderen Analyseebene zu betrachten. Man kann eine Präferenz für Wörter mit Geminaten aufstellen, nach der mehr konsonantische Stärke in der Geminate besser ist. Diese neue Präferenz auf der Ebene des Wortes kann man dann durch weitere Lautwandel belegen: zum Beispiel durch die Entwicklung von /nt/ und /nk/ $\mathrm{zu} / \mathrm{tt} / \mathrm{und} / \mathrm{kk} / \mathrm{im}$ Altnordischen. Bei Gegenevidenz, z. B. der Entwicklung von $/ \mathrm{np} /$ und $/ \mathrm{lp} / \mathrm{zu} / \mathrm{nn} /$ und $/ 11 /$ in derselben Sprache, weicht man wieder auf die Ebene der Assimilation der konsonantischen Stärke in heterosyllabischen Clustern aus. So kommt man zum Schluss, dass Lautwandel, die Strukturen nach einem bestimmten Parameter verändern, in allen Sprachen prinzipiell in die gleiche Richtung gehen, trotz der Entwicklung des heterosyllabischen Clusters /rl/ bald $\mathrm{zu} / \mathrm{rr} /$ und bald $\mathrm{zu} / 11 /$.

In diesem Punkt bleibt die Präferenztheorie klar hinter anderen teleologischen Lautwandelkonzepten zurück, die mit universellen Tendenzen operieren. So arbeitet z. B. auch die Optimalitätstheorie mit einem Satz universeller Constraints, die durch andere Constraints aufgehoben bzw. überschrieben werden können. ${ }^{13}$ Viele Forscher sehen in diesen Constraints - die oft ebenfalls aus Lautwandeln gewonnen werden - ein Äquivalent der universellen Präferenzen (vgl. die Übersicht bei Ritt 2001: 294-297, außerdem z. B. Wurzel 1999: 242-243, Dziubalska-Kołaczyk 2001: 71-77, Gibbon 2001: 185-194). Allerdings sind die optimalitäts-

12. Ausführlich zu diesem Punkt z. B. Murray (2000: 228-32).

13. Grundlegend zur Theorie jetzt vor allem Kager (1999) und Prince \& Smolensky (2004); zur Anwendung auf die Lautwandelproblematik vgl. z. B. Ham (1998), Gess (2003) mit Lit. und die bei Holt (2003) gesammelten Fallstudien; kritisch zu ihren Ergebnissen z. B. Loporcaro (2001, 2007), McMahon (2003) und Blevins (2004, 2006). 
theoretischen Constraints in einer konkreten Sprache hierarchisch organisiert: ein Constraint kann nur durch höhere Constraints aufgehoben bzw. überschrieben werden, nicht durch beliebige Constraints. Lautwandel entstehen dabei im Prinzip als Ergebnis von Änderungen in solchen einzelsprachlichen Constrainthierarchien. Die hierarchische Organisation des Constraintinventars schränkt die Erklärungsmöglichkeiten bei der Behandlung des empirischen Materials nicht unerheblich ein. So führt z. B. die Verbindung der Entwicklung von $/ \mathrm{np} / \mathrm{zu} / \mathrm{nn} / \mathrm{im}$ Altnordischen mit $\mathrm{Zu}$ - oder Abnahme der konsonantischen Stärke im theoretischen Rahmen der Optimalitätstheorie - anders als in dem der Präferenztheorie - zu Problemen, weil /nt/ und /nk/ sich dann nicht zu /tt/ und $/ \mathrm{kk} /$ entwickeln dürften.

- Zweitens finde man bei der Untersuchung synchroner Befunde von Sprachen, dass sie mehr Strukturen enthalten, die nach Auskunft der Lautwandel relativ viel Qualität besitzen. ${ }^{14}$ Dies bestätige Präferenzen, die aus den Lautwandeln gewonnen werden.

Das Argument ist prinzipiell identisch mit dem ersten, weil Vennemann selbst synchrone Befunde dieser Art auf einschlägige Lautwandel zurückführt.

- Drittens gebe es für die ermittelten Präferenzen Bestätigungen aus den Nachbardisziplinen, nämlich der Soziologie, der Kommunikationstheorie, der Theorie des Erstspracherwerbs, der Phonetik und einigen anderen.

Als Beispiel für eine solche Bestätigung bringt Vennemann die mündliche Versicherung nicht namentlich genannter Phonetiker, dass zum Beispiel /kl-/ im Anlaut weniger phonetisch komplex, somit leichter auszusprechen sei als /kn-/, wobei die einschlägige Präferenz /kl-/ mehr Qualität zuspricht als $/ \mathrm{kn}-/$.

Der Begriff „Bestätigung“ impliziert einen nachgewiesenen Zusammenhang zwischen dem, was bestätigt wird, und dem, was es bestätigt. Dies ist selbst dann der Fall, wenn es sich um Daten verschiedener Diszi-

14. In Vennemanns (1993: 326) eigenen Worten: „A language system will in general not contain a structure on a given parameter without containing those structures constructible with the means of the system that are more preferred in terms of the relevant preference law“. Allerdings ,discontinuities occasionally do arise on a given parameter, though only as a consequence of changes that are not directed at that particular parameter but only involve it incidentally, such as ... a change on a different parameter that happens to intersect the given parameter". 
plinen handelt. Das archäologisch nahe liegende hohe Alter einer Mumie kann durch die Messung ihres $\mathrm{C}^{14}$-Gehalts bestätigt werden, weil fest steht, dass die Menge von $\mathrm{C}^{14}$ in organischen Stoffen mit der Zeit abnimmt. Man kann dagegen im metallenen Glanz des Goldes keine Bestätigung seiner niedrigeren Valenz im Vergleich zum nicht glänzenden Schwefel sehen, außer es ist nachgewiesen, dass Glanz, aus welchem Grund auch immer, normalerweise von niedriger Valenz begleitet wird. Die angesprochene Korrelation zwischen einer der ermittelten Präferenzen und dem Grad der phonetischen Komplexität der betreffenden Struktur wäre somit nur dann eine Bestätigung, wenn zwischen der Qualität einer phonologischen Struktur im Sinne der Präferenztheorie und dem Grad der phonetischen Komplexität dieser Struktur ein anderweitig nachgewiesener Zusammenhang bestehen würde. Solange das nicht der Fall ist, kann man nicht von einer Bestätigung sprechen. ${ }^{15}$

Alle drei Argumente, die Vennemann gegen den Zirkelschlusscharakter seines Verfahrens bei der Ermittlung der Präferenzen aus den einschlägigen Lautwandeln anführt, können somit leicht entkräftet werden.

Welchen Status haben die aus den Lautwandeln gewonnenen Präferenzen in Wirklichkeit? Die Antwort auf diese Frage ist einfach: Strukturen, die nach der Präferenztheorie mehr Qualität besitzen, sind in Wirklichkeit Strukturen, die durch das Wirken verschiedener Lautwandel relativ häufig entstehen. Nachdem verschiedene Arten von Konsonantenschwund und Vokalepenthese automatisch zu Silben mit leichterem Auslaut führen, ohne notwendigerweise auf der Ebene der Silbe motiviert zu sein, ergibt sich eine Präferenz für leichteren Auslaut der Silbe. Es ist klar, dass die Zuweisung eines konkreten Lautwandels in einer Sprache zu einer solchen Präferenz nicht als Erklärung dieses Lautwandels aus den fundamentalen Eigenschaften der menschlichen Sprache gelten kann, also keine Erklärung im Sinne der Präferenztheorie ist.

Aus dem Gesagten ergibt sich folgendes Fazit. Die Theorie der phonologischen Präferenzen hat das Potential, das von der traditionellen jung-

15. Von einer direkten Verbindung der präferenztheoretischen Qualität mit der phonetischen Komplexität (vgl. etwas vage bereits Vennemann 1988: 67) wäre übrigens abzuraten. Eine solche Verbindung würde implizieren, dass die Zunahme der Qualität bei jedem Lautwandel notwendigerweise mit der Abnahme der phonetischen Komplexität einhergehen muss. Wenn man diese Annahme macht, nimmt man der Präferenztheorie die Möglichkeit, viele Lautwandel zu erklären, die mit Zunahme der phonetischen Komplexität auf der betreffenden Ebene der phonologischen Struktur verbunden sind. Zum Beispiel nimmt die phonetische Komplexität bei der Entwicklung von /w-/ zu / $\overparen{g w}-/$ im britannischen Keltisch und einigen romanischen Sprachen sowohl in Bezug auf das Konsonantenphonem selbst als auch auf der Ebene der betreffenden Silbe zu. Wenn die Präferenztheorie solche Lautwandel grundsätzlich nicht zu erklären vermag, ist sie für die historische Phonologie kaum von Nutzen. 
grammatischen Forschung Erreichte zu vertiefen, und zwar durch die Aufdeckung der Motivation eines konkreten Lautwandels in einer konkreten Sprache. Ob das tatsächlich gelingt, hängt allerdings von den folgenden zwei Voraussetzungen ab. Erstens müssen die universellen phonologischen Präferenzen zuverlässig ermittelt werden. Zweitens muss sich die Vermutung bestätigen, dass jeder Lautwandel prinzipiell zur $\mathrm{Zu}$ nahme der Qualität führt. Bis jetzt ist weder das eine noch das andere geschehen.

\section{Lautwandel als Tendenzen}

Empirische Arbeit an konkreten Problemen in der historischen Phonologie vor allem der germanischen Sprachen brachte einige Vertreter der Präferenztheorie zu der Auffassung, dass das bekannte junggrammatische Postulat von der ausnahmslosen Geltung der Lautgesetze auf dem Hintergrund der neuen Erkenntnisse in seiner ursprünglichen Form nicht zu halten ist und daher modifiziert werden muss. In Vennemann (2000) wird vorgeschlagen, in den Lautwandeln der germanischen Einzelsprachen prinzipiell nur Tendenzen zu sehen, denen in jedem konkreten Fall alle einschlägigen Lautstrukturen in der betreffenden Sprache folgen können, aber nicht müssen. Wenn präferenztheoretische Optimierung einer Lautstruktur durch unterschiedliche Lautwandel erreicht werden kann, komme es nach Vennemann durchaus vor, dass auch innerhalb einer Sprache unter identischen Bedingungen gleich zwei oder mehr Lautwandel mit gleichem Ausgangspunkt, aber unterschiedlichen Ergebnissen wirken. Diese Hypothese Vennemanns wird im vorliegenden Abschnitt anhand der beigebrachten empirischen Evidenz eingehend überprüft. Die Überprüfung zeigt, dass die vorgeschlagene Modifikation des junggrammatischen Postulats von der Ausnahmslosigkeit der Lautgesetze, folglich von der regelmäßigen Wirkung der durch diese Lautgesetze implizierten Lautwandel, weder aus den Grundannahmen der Präferenztheorie folgt, noch durch das herangezogene empirische Material nahe gelegt wird.

Der Ausgangspunkt der Überlegungen Vennemanns (2000) ist die Entwicklung der alten vorgermanischen Konsonantencluster vom Typ /sKR-/ (K = Velar, $\mathrm{R}=$ Resonant) im Wortanlaut verschiedener germanischer Sprachen. Vennemann nimmt im Anschluss an ältere Forschung an, dass der Konsonantencluster ${ }^{*} \mathrm{KKl}$, in vorgermanischer Zeit im Wortanlaut noch möglich, in den historischen germanischen Sprachen in allen betreffenden Fällen vereinfacht wurde. Die Evidenz, auf die sich Vennemann stützt, bilden in erster Linie Dubletten vom Typ ahd. glìtan, ae. glīdan ,gleiten' neben mhd. slītan, ae. slüdan ,gleiten'. Die sehr ähnliche Lautung und identische Semantik der beiden Verben legen nahe, 
dass es sich um Varianten ein und desselben Wortes handelt. Dabei ist ein Wechsel $*^{*}-\sim *_{s}$ - außerhalb des Germanischen nicht bekannt: er wäre hochgradig erklärungsbedürftig. Gut bekannt, wenn auch nicht verstanden, ist dagegen eine Erscheinung, die man traditionell „bewegliches $s^{\text {" }}$ nennt. In verschiedenen älteren indogermanischen Sprachen, darunter auch dem Urgermanischen, findet man nämlich relativ oft ein und dasselbe Wort in zwei Varianten, die sich voneinander lediglich durch An- bzw. Abwesenheit eines * ${ }^{s}$ - im Anlaut unterscheiden. Wenn man nun mit Vennemann versucht, auch die Dubletten ahd. glìtan, ae. glīdan mhd. slìtan, ae. slìdan ,gleiten" dem Phänomen des ,,beweglichen s“ zuzuordnen, bekommt man für die vorgermanische Zeit $*^{h} g^{h} l e i d^{h} e$ - neben ${ }^{*} s^{h} l e i d^{h} e$-. Die Variante vorgerm. ${ }^{*} g^{h} l e i d^{h} e$ - würde direkt zu ahd. glìtan, ae. glīdan führen. Wenn man annimmt, dass der schwere Konsonantencluster im Anlaut der Variante vorgerm. ${ }^{*}{ }^{\prime} g^{h} l e i d^{h} e$ - durch den Schwund des Velars erleichtert wurde, bekommt man mhd. slìtan, ae. slìdan. Auf die Annahme eines rätselhaften Wechsels urgerm. ${ }^{*} g_{-} \sim *_{s-}$ kann bei dieser attraktiven Analyse verzichtet werden.

Vennemann deutet die Vereinfachung des alten ${ }_{s} \mathrm{Kl}$ - in den germanischen Sprachen im Sinne der Präferenztheorie: Lautgruppen dieser Art sind relativ schlecht, sie werden in vielen Sprachen abgebaut, so auch im Germanischen. Dabei gibt es allerdings mehr Möglichkeiten, aus dem schlechten Cluster /sK1-/ ein präferenztheoretisch besseres zu machen. Die Entwicklung von /sKl-/ zu /sl-/, die in den gerade besprochenen Fällen vorliegt, ist nur eine davon. Eine weitere Möglichkeit besteht zum Beispiel in der Entwicklung von /sKl-/ zu /sK-/, wobei nicht der Velar, sondern die Liquida schwindet. Vennemann nimmt an, dass auch diese Entwicklung für die germanischen Sprachen postuliert werden kann. Das alte ${ }_{s} K l$ - kann sich in den einen Lexemen einer germanischen Sprache $\mathrm{zu} * s l$ - entwickelt haben, in den anderen dagegen $\mathrm{zu} * s K$-. Die Lautwandel ${ }_{s} \mathrm{Kl}$ - $>*_{s l}$ - und ${ }^{s} \mathrm{SKl}->{ }_{s K} \mathrm{~K}$ - sind Tendenzen, die in ein und derselben Sprache unter identischen Bedingungen beide möglich sind, weil sie gleichermaßen zu mehr Qualität im Sinne des einschlägigen Parameters führen. Das junggrammatische Postulat von der ausnahmslosen Wirkung der Lautwandel unter gleichen Bedingungen sei aufzugeben.

Die Überlegenheit seiner Position über die junggrammatische Forschung versucht Vennemann anhand des folgenden Falls zu erweisen.

Der Lautwandel vorgerm. ${ }^{*} \mathrm{sKl}$ - > urgerm. ${ }^{*} \mathrm{sl}$ - wurde von der traditionellen Forschung aufgestellt, um Fälle vom Typ ahd. sliozan, afr. slūta $<$ urgerm. *sleute- ,schließen' neben offensichtlich verwandtem lateinisch claudō ,schließen' zu erklären (vgl. z. B. Seebold 1970: 436-437, Vine 1985: 67). Wenn man den Anlaut des urgermanischen Verbs auf älteres *skl- zurückführt, also ein vorgerm. *skleude- rekonstruiert, gelangt man auch hier zu zwei Varianten, die sich voneinander im Allge- 
meinen durch die An- oder Abwesenheit eines ${ }^{*} s$ - unterscheiden: vorgerm. *skleude- und vorlat. *klaude- ,schließen' (der Unterschied im Vokalismus kann sekundär sein). Soweit die traditionelle Forschung.

Neben urgerm. *sleute-, schließen', das durch ahd. sliozan und afr. slüta vorausgesetzt wird, kennt das Urgermanische noch ein schwaches Verb *skutjan-, dessen Fortsetzungen in den historischen germanischen Sprachen eine ähnliche Semantik aufweisen: vgl. ae. scyttan, das in etwa ,schließen' bedeutet haben kann. Vennemann nimmt an, dass urgerm. *sleute- ,schließen' und *skutjan- mit ähnlicher Semantik etymologisch verwandt sind, indem sie beide von der vorgermanischen Wurzel für ,schließen' mit ihrem *skl- im Anlaut gebildet sind. Der relativ schlechte Cluster vorgerm. *skl- wurde dabei in urgerm. *sleutan- durch den Schwund des Velars verbessert, in urgerm. *skutjan- durch den Schwund der Liquida. Für die traditionelle Forschung, die sich auf das Postulat der ausnahmslosen Wirkung der Lautwandel stützt, sind urgerm. *sleutan- und *skutjan- dagegen trotz der ähnlichen Lautung und ähnlichen Semantik nicht verwandt, weil sich vorgerm. ${ }^{*} s k l$ - unter gleichen Bedingungen nicht bald zu*sl-, bald zu*sk- entwickelt haben kann. Die Position Vennemanns sei somit der traditionellen Forschung überlegen, weil sie es ermöglicht, die etymologischen Verhältnisse, in denen sich urgerm. *sleutan- ,schließen' befindet, vollständiger zu erfassen.

Hier muss zunächst klargestellt werden, dass die Annahme Vennemanns, bei Lautwandeln handele es sich um Tendenzen, in keiner Weise aus den Grundannahmen der Präferenztheorie folgt. Wenn man zum Beispiel feststellt, dass /sKl-/ ein relativ schlechter Cluster ist, /sl-/ und /sK-/ dagegen besser, ist im Rahmen der Präferenztheorie lediglich davon auszugehen, dass /sKl-/ zu /sl-/ oder /sK-/ werden kann. Darüber, ob unter identischen Bedingungen in ein und derselben Sprache gleich beide Möglichkeiten der Entwicklung von /sKl-/ realisiert werden können oder nicht, macht die Präferenztheorie keine Aussage. Es ist durchaus möglich, den Grund für den Abbau von /sKl-/ mit der Präferenztheorie in der relativ geringen Qualität dieses Clusters zu sehen, den Grund für seine Ersetzung ausgerechnet durch /sl-/ oder /sK-/ entsprechend in der besseren Qualität dieser Lautgruppen, die Ersetzung selbst aber als klassischen Lautwandel aufzufassen, der unter gleichen Bedingungen in ein und derselben Sprache entweder immer zu /sl-/ oder immer zu /sK-/ führt.

Die von Vennemann aufgeworfene Frage danach, ob Lautwandel regelmäßig wirken oder statistische Tendenzen sind, steht somit zur Präferenztheorie in keiner erkennbaren Beziehung und ist unabhängig davon zu beantworten, ob diese Theorie stimmt oder nicht. Bei dieser Frage handelt es sich auch in der Tat nicht um eine Errungenschaft der präferenztheoretischen ,post-neogrammarian“ Zeit, wie Vennemann irrtüm- 
lich glaubt (vgl. besonders 2000: 254-256), sondern um die klassische Kontroverse zwischen der junggrammatischen Richtung in der historischen Phonologie und ihren Vorgängern. Vennemann bezieht in dieser Kontroverse eine Position, die man forschungsgeschichtlich eher „preneogrammarian" nennen müsste. ${ }^{16}$

Um die Argumente Vennemanns gegen die regelmäßige Wirkung der Lautwandel richtig zu bewerten, muss man sich zuerst in Erinnerung rufen, wie die Forschung zur Annahme ihres regelmäßigen Charakters kam, d.h. aus welchen Gründen das Postulat von der ausnahmslosen Wirkung der Lautgesetze aufgestellt wurde.

In der Frühphase der historischen Sprachwissenschaft - zu Grimms, Bopps und Schleichers Zeit - sah man in den Lautwandeln Tendenzen, die nicht notwendigerweise alle einschlägigen Fälle in einer Sprache erfassen mussten. Zum Beispiel entsprach dem Laut $t$ des Griechischen, Lateinischen oder Altindischen in germanischen Sprachen in vielen Fällen der stimmlose Spirant $b$, so dass man mit einem Lautwandel altes * $t$ $>$ urgerm. ${ }^{*} b$ rechnen konnte. Es waren aber auch Lexeme bekannt, bei denen dem $t$ der anderen indogermanischen Sprachen im Germanischen ein $t$ entsprach, so dass sich ein Lautwandel altes $* t>$ urgerm. ${ }^{*} t$ ergab, bei dem sich streng genommen nichts änderte. Schließlich kannte man auch Fälle, die einen Lautwandel altes $* t>$ urgerm. $* \partial$ nahelegten. Darin, dass sich altes indogermanisches $* t$ im Urgermanischen bald überhaupt nicht änderte, also $* t$ blieb, bald zu $* b$ und bald zu * ${ }^{*}$ wurde, sah man keinen Widerspruch, weil die Lautwandel nur Tendenzen waren. Die immer bessere Erforschung des Materials führte allerdings zur Beobachtung, dass jedem der genannten Lautwandel - idg. ${ }^{*} t>$ urgerm. ${ }^{*} p$, idg. ${ }^{*} t>$ urgerm. ${ }^{*} t$ und idg. ${ }^{*} t>$ urgerm. ${ }^{*} \delta-$ ein eigener Wirkungsbereich zukam, in dessen Grenzen der betreffende Lautwandel fast ausnahmslos durchgeführt wurde. Idg. ${ }^{*} t>$ urgerm. ${ }^{*} t$ beobachtete man immer nach $s$ oder Plosiven, wo die Entwicklung zu $* b$ oder $* \delta$ nicht vorkam. Idg. ${ }^{*} t>$ urgerm. ${ }^{*} \partial$ war ausschließlich im Inlaut nach unbe-

16. Daran ändert auch die Tatsache nichts, dass Lautwandel, die ein und dieselbe Struktur nach dem gleichen Parameter optimieren, bei Vennemann wohl immer sämtliche Vorkommen dieser Struktur erfassen. Die Vorgänger der Junggrammatiker arbeiteten ebenfalls häufig mit Lautwandeln, die alle Fälle in ihrem Wirkungsbereich erreichten, und dennoch nur Tendenzen waren, weil sich das Ursprüngliche nicht notwendigerweise überall gleich veränderte. So wurde z. B. der bekannte slavische Wechsel $k \sim c \sim \check{c}-$ z. B. in aksl. Gen. Sg. vlŭka Lok. Sg. vlücě $\sim$ Vok. Sg. vlǔče ,Wolf ${ }^{‘}-$ auch damals als Palatalisierung der Velare vor hellen Vokalen erklärt. Diese Tendenz erfasste auch alle einschlägigen Fälle. Warum aber aksl. $k$ bald zu $c$ palatalisiert wurde und bald zu $\check{c}$, war im vorjunggrammatischen Forschungsparadigma vernachlässigbar. So werden auch bei Vennemann alle Vorkommen von vorgerm. ${ }^{*} s k l$ - modifiziert, meist zu ${ }^{*} s l$-, manchmal aber zu *sk-, ohne dass es für den Unterschied einen Grund gäbe. 
tonter Silbe bezeugt, deckte aber alle Fälle dieser Art ab. Idg. ${ }^{*} t>$ urgerm. ${ }^{*} b$ beobachtete man im Anlaut und im Inlaut nach betonter Silbe, die Stellung nach $s$ oder Plosiven ausgenommen. Bei den wenigen Ausnahmen aus der festgestellten Verteilung handelte es sich ausschließlich um Nomina mit der Entwicklung idg. ${ }^{*} t>$ urgerm. ${ }^{*} t$ anstelle von ${ }^{*} p$ oder $* \partial$, wie zum Beispiel bei lat. tēgula ,Dachziegel ${ }^{*} \sim$ ae. tigele, as. tēgala ,Ziegel‘. Die Semantik sowie andere lautliche Charakteristiken dieser Wörter legten die Annahme rezenter Entlehnungen aus dem Lateinischen nahe. So führte die genaue Untersuchung des Materials zu der empirischen Feststellung, dass die Lautwandel idg. ${ }^{*} t>$ urgerm. ${ }^{*} b$, idg. ${ }^{*} t>$ urgerm. ${ }^{*} t$ und idg. ${ }^{*} t>$ urgerm. ${ }^{*} \partial$ keine Tendenzen waren, die in einem konkreten Fall wirken konnten oder auch nicht, sondern Prozesse, die im jeweiligen Wirkungsbereich ausnahmslos alle einschlägigen Fälle erfassten. Man beachte, dass der Übergang von den unverbindlichen Tendenzen zu den ausnahmslosen Lautwandeln eigentlich in einer präziseren Beschreibung des einschlägigen empirischen Materials besteht.

Genauso durch eingehende Untersuchung des vorliegenden empirischen Materials wurde festgestellt, dass es sich auch bei anderen Lautwandeln in anderen Sprachen nicht um Tendenzen handelt, die miteinander konkurrieren können, sondern um ausnahmslose Lautgesetze: idg. $* k^{w}$ wurde im Vedischen zur Affrikate $c$ vor hellen Vokalen, zum Plosiv $k$ vor dunklen Vokalen; urgerm. ${ }^{*} e$ wurde im Altnordischen zu $i a$ vor $* a$ in der nächsten Silbe, zu io vor $* u$ in der nächsten Silbe; altrussisch $e$ wurde zu russ. $o$ unter Akzent, zum hellen Schwa unter Nichtakzent usw. Auf der Grundlage unzähliger Befunde dieser Art wurde geschlossen, dass die Lautwandel generell ausnahmslos wirken. Konkurrierende Tendenzen sind dabei regelmäßige Lautwandel unter unterschiedlichen Bedingungen.

Das Prinzip der regelmäßigen Wirkung der Lautwandel ist somit kein theoretisches Konstrukt, sondern es beruht auf genauer Beobachtung empirischer Daten. ${ }^{17}$ Der Einwand Vennemanns besteht nun darin, dass

17. Die Auffassung des junggrammatischen Postulats von der regelmäßigen Wirkung der Lautwandel als eine rein theoretische Annahme, die am empirischen Material prinzipiell nicht überprüft werden kann, ist leider ein sehr verbreitetes Missverständnis. Die Quelle dieses Missverständnisses besteht wohl darin, dass die junggrammatische Forschung die Ausnahmen aus den festgestellten Regelmäßigkeiten beim Lautwandel häufig durch die Annahme von Entlehnung oder analogischem Ausgleich zu erklären versucht. Daraus zieht man den falschen Schluss (so explizit z. B. Wang 1969: 9-10, Lieberman 1991: 311-312, Wang \& Lien 1993: 353), dass gegen die regelmäßige Wirkung der Lautwandel generell keine Evidenz beizubringen sei, weil diese jederzeit durch Entlehnung oder Analogie wegerklärt werden könne. In Wirklichkeit werden Entlehnung oder Analogie in der traditionellen junggrammatischen Forschung nicht nach Belieben bemüht, um jede Ausnahme zu umgehen, sondern sind in jedem konkreten Fall besonders zu begründen. Bei Entlehnungen ist generell das konkrete Lexem in einer konkreten Gebersprache 
er auf einen konkreten Fall hinweisen kann, bei dem altes *skl- in ein und derselben Sprache bald zu*sl-, bald zu*sk- zu werden scheint. Die Stärke eines solchen Arguments hängt grundsätzlich davon ab, wie sicher die etymologische Zusammenstellung der betreffenden Lexeme in der betreffenden Sprache ist. Im vorliegenden Fall muss man sich fragen, ob die Herleitung von urgerm. *sleutan- und *skutjan- aus einer Quelle zweifellos zutrifft oder plausible Alternativen vorhanden sind. Dies soll nun in aller Kürze untersucht werden.

Die genaue Semantik von urgerm. *skutjan- ist nicht ganz geklärt. Die altenglische Fortsetzung des Verbs scyttan ist nur einige wenige Male belegt. Der älteste Beleg findet sich bei Ælfric ic scytte sum loc oððe hapsige ,ich scytte einen Bolzen oder mache fest' (ÆGram 219.15). ${ }^{18}$ Das Syntagma glossiert lat. sero seras ,ich füge die Querriegel ein', so dass ic scytte sum loc am ehesten etwa ,ich füge einen Riegel ein' bedeutet. Ganz ähnlich scheint das Verb verwendet in einem weiteren Beleg: hē gelahte ðā dura and ne mihte ðā scyttelsas unscyttan swā hraðe, er packte das Tor, konnte aber die scyttelsas nicht gleich un-scyttan (ÆLS Martin 860). Das Objekt von un-scyttan ist hier das von dem Verb selbst

zu nennen, und es ist wahrscheinlich zu machen, dass aus eben dieser Sprache Lexeme mit gegebener Semantik entlehnt werden konnten. Die bloße Behauptung, das Lexem mit lautlicher Abweichung könne auch ein Lehnwort sein, ist noch keine Erklärung im junggrammatischen Sinne (vgl. die Richtigstellung bei Kiparsky 1988: 370-372). Bei Analogien ist stets das Muster für die analogische Neuerung anzugeben und zu zeigen, wie die Ersetzung der belegten oder hypothetischen regulären Form durch die geneuerte stattgefunden hat. Die bloße Behauptung, eine konkrete Abweichung könne auch analogisch bedingt sein, ist wiederum noch keine Erklärung. Dieses Vorgehen hat zur Folge, dass bei weitem nicht jede denkbare Abweichung von den festgestellten Regelmäßigkeiten im junggrammatischen Forschungsparadigma erklärbar ist. Würde man zum Beispiel - um bei der im Haupttext benutzten Entwicklung des alten $* t$ im Germanischen zu bleiben - im Urgermanischen wurzelverwandte Lexeme entdecken, die im Wortanlaut vor gleichem Vokal teils das erwartete $* p$, teils das sonst nur im Inlaut mögliche $* \partial$ aufwiesen, wäre das Postulat der regelmäßigen Wirkung der Lautwandel widerlegt, weil Lexeme mit $* \partial$ weder entlehnt noch analogisch entstanden sein könnten. Die Abwesenheit klarer Fälle dieser Art in der Empirie der bisher untersuchten Sprachen ist das eigentliche Argument für das regelmäßige Wirken der Lautwandel. Bei den oft kritisierten Aussagen der Begründer der junggrammatischen Forschungsrichtung zur Rolle des Individuums beim Lautwandel usw. handelt es sich demgegenüber um Versuche, die aus der Empirie gewonnene Erkenntnis theoretisch zu interpretieren. Kritik an diesen Versuchen kann das regelmäßige Wirken der Lautwandel nicht widerlegen. Dass die Regelmäßigkeit logisch betrachtet nicht die einzige Möglichkeit ist (Schuchardt 1885, nach ihm z. B. Wilbur 1972 und Vennemann 1972), ist richtig, aber genausowenig weiterführend, wie zum Beispiel die Feststellung, dass sich die Planeten des Sonnensystems logisch besehen nicht notwendigerweise auf Ellipsenbahnen zu bewegen brauchen, weil man im Prinzip auch an Kreise denken kann.

18. Altenglische Belege werden zitiert nach dem elektronischen Dictionary of Old English Corpus. 
abgeleitete Nomen scyttels, das an anderer Stelle, ebenfalls im Plural, lat. serae ,Querriegel' glossiert. Zu übersetzen ist somit etwa ,er packte das Tor, konnte aber die Riegel nicht gleich herausziehen'. Vgl. schließlich noch den Glossenbeleg (HyGl3 Gneuss 102.3) we biddað unbind ūs fram eallum synnum mid häse ge ðe belūcað heofonan mid worde and geunscyttað hire scyttelsas gl. quesumus soluite nos ab omnibus peccatis cum iussu, qui clauditis celum cum uerbo soluitisque seras eius, Wir bitten, befreit uns mit (eurem) Befehl von allen Sünden, die ihr den Himmel verschließt, und durch (euer) Wort seine Querriegel löst'.

Außerdem verfügt man über zwei Belege für for-scyttan aus den altenglischen Homilien. Hier ist das Verb etwa im Sinne von ,hindern' gebraucht: hī hêfodon folces synna and heora wrace on him sylfum forscytton, sie beklagten die Sünden der Menschen und verhinderten ihre Rache an ihnen selbst' (ÆCHom I,36 487.48), „at ðā sceortan wītu ðises geswincfullan līfes forscyttan ðā toweardan ðē nā́fre ātēoriað ,dass die kurzen Torturen dieses qualvollen Lebens diejenigen hindern, zu kommen, die unfehlbar sind' (ÆCHom II,21 188.259). Vgl. ferner die eindeutigen Glossenbelege forscyttende gl. obdens .i. opponens ,entgegensetzend“ (PrudGl 1 Meritt 662) und min god mildheortnys his forscytte 1 forestepp $ठ$ me gl. deus meus misericordia eius praeueniet me (PsGl I Lindelöf 58.11) ,mein Gott, seine Gnade soll mir zuvorkommen'.

Die altfriesische Fortsetzung von urgerm. *skutjan- lautet sketta. Das Verb ist nur zweimal bezeugt, im Objekt steht einmal wetter-gongen ,Wasserabzüge' und einmal blōd ,Blut'. Afr. sketta bedeutet somit ,(Wasserabzug) schließen', ,(Blutfluss) stoppen'. Ganz ähnlich wird mittelniederdeutsch und mittelniederländisch schutten, mittelhochdeutsch schützen verwendet: es bezieht sich bei nichtmetaphorischem Gebrauch auf Wasser und bedeutet etwa ,eindämmen', ,aufstauen', woraus sich sekundär die Semantik ,abwehren', ,hindern' entwickelt, aber auch ,einfrieden', ,einpferchen', ,einschließen'.

Auf dem Festland bedeuten die Fortsetzungen von urgerm. *skutjansomit ,Flüssigkeiten am Fließen hindern'. Diese Semantik muss auch für ae. for-scyttan angenommen werden: sonst versteht man den metaphorischen Gebrauch als ,(Rache) verhindern', ,(Menschen am Kommen) hindern' nicht. Eine direkte Spur der Bedeutung ,eindämmen', ,aufstauen findet man möglicherweise in der Verwendung des präteritalen Partizips scytt bei Beschreibung von Landschaften in altenglischen Urkunden, wo es ae. brōc ,Bach', mere ,See', dūn ,Heideland, Moor' und facnn ,Sumpf ' charakterisieren kann. Daneben bedeutet ae. scyttan nach Ausweis der oben besprochenen Belege etwa ,(Bolzen, Riegel) einfügen', mit un,(Riegel) herausziehen'. Aus diesem Befund ergibt sich für urgerm. *skutjan- als ursprüngliche Bedeutung etwa ,einschieben' im Sinne von ,(Riegel ins Schloss) einfügen', aber auch als ,(Bach) eindämmen', ,(Wasser- 
strahl) zustopfen'. Aus dieser Semantik entwickelt sich auf der Insel sekundär die Bedeutung ,schließen', die man bei dem schwachen Verb seit der mittelenglischen Zeit belegt.

Die Semantik ,einschieben' ist nun nicht weit entfernt von der Bedeutung des urgermanischen starken Verbs, mit dem *skutjan- in der traditionellen Forschung immer zusammengestellt wurde. Urgerm. * skeutanbedeutet überall, wo es fortgesetzt ist, ,schießen'. Im Altwestnordischen wird seine Fortsetzung aber auch im Sinne von etwa ,schieben' verwendet: Brücke über einen Graben, Schiff ins Wasser, Kiste mit einem Toten übers Bord, schließlich auch Riegel vor die Tür (awn. skiōta loku fyrir $h u r ð u$,einen Riegel vor die Tür schieben'). Diese Verwendung findet man auch bei der altenglischen Fortsetzung von *skeutan-. Man vgl. he lēt dragan üp ðane dēadan Harald and hine on fen scēotan, er ließ den toten Harald hinaufziehen und ihn ins Moor stoßen' (ChronC 1040.7). Ferner

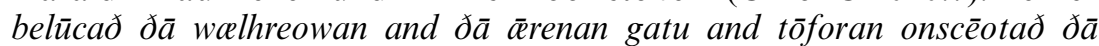
ysenan scyttelsas ,sperrt ein die Grausamen und (sperrt zu) das eherne Tor, zuvor aber schiebt ein die eisernen Riegel!' (Nic A 21.1.9). Schließlich noch hē arn swīðe of stlīce and ðā cyrican belēac and mid scyttelum bescēat and gefastnode, er eilte sofort schnell (dorthin), sperrte die Kirche ab, beschob und befestigte (sie) mit Riegeln' (GDPref C 29.234.17).

,Schießen` kann sich leicht über ,schleudern' aus ,schieben' entwickelt haben. ,Schieben' aus ,schießen' ist dagegen schwierig. Aus diesem Grund ist die ursprüngliche Bedeutung von urgerm. * skeutan- am ehesten etwa ,schieben'. Dies legt etymologische Verwandtschaft mit urgerm. *skutjan- ,einschieben' nahe. Die Verben stehen einander semantisch so nahe, dass sie im Altenglischen mit demselben Objekt scyttelsas, Querriegel' $^{`}$ zur Bezeichnung desselben Vorgangs verwendet werden konnten: vgl. die bereits zitierten Belege ne mihte dā scyttelsas unscyttan ,konnte die Querriegel nicht herausziehen' und onscēotað dā yssenan scyttelsas ,schiebt ein die eisernen Querriegel!'.

Die von Vennemann bevorzugte Zusammenstellung von urgerm. *skutjan- ,einschieben' mit *sleutan-, schließen' ist in semantischer Hinsicht natürlich ebenfalls möglich. Sie ist aber angesichts einer plausiblen Alternative nicht zwingend und kann deshalb nicht als Argument gegen den grundsätzlich regelmäßigen Charakter der Lautwandel gelten. ${ }^{9}$

19. Vennemann legt besonderen Wert auf die morphologische Ähnlichkeit der Nomina ae. scyttel, das offensichtlich mit *skutjan- zusammenhängt, und ahd. sluzzil, as. slutil, die von *sleute- nicht zu trennen sind. Er nimmt an, dass es sich um zwei Fortsetzungen ein und desselben Nomens handelt, wobei der Anlautcluster *skl- im Altenglischen zu *sk-, auf dem Kontinent zu *sl- wurde. Die auffällige Gemination in ae. scyttel ist nach Vennemann Ergebnis einer sekundären Anlehnung an das Verb ae. scyttan $<*$ skutjan-. Man muss allerdings festhalten, dass die Nomina nicht dieselbe Semantik haben. Ae. scyttel entspricht nach Ausweis der Glossenbelege lat. sera "Querriegel' und uectis ,Sperrbalken': vgl. he gestrangode scyttelas gata ðīnra gl. confortauit seras portarum tuarum, verstärkte die Querriegel deines Tores' (PsGlG Rosier 147.2) und geðraste geatu 
Vennemann (2000) bringt noch einen weiteren Grund anzunehmen, dass durch Präferenzen hervorgerufene Lautwandel nicht regelmäßig wirken, sondern nur Tendenzen sind. Er weist auf einen klaren Fall hin, bei dem ein Anlautcluster mit relativ geringer Qualität in ein und demselben Wort in derselben Sprache, also unter identischen Bedingungen, bald aufgelöst wird, bald aber bestehen bleibt. Dieses Wort ist das starke Verb mit der Bedeutung,sprechen', das traditionell als urgerm. *sprekan- rekonstruiert wird: vgl. altsächsich sprekan und altfriesisch spreka ,sprechen'. Das Verb kommt nur in westgermanischen Sprachen vor. In einigen davon erscheint es dabei in zwei Varianten: einer mit spr- und einer mit einfachem $s p$ - im Anlaut. Die betreffenden Sprachen sind das Altenglische mit sprecan in den anglischen Quellen neben mehrmals bezeugtem specan in den westsächsischen, das Althochdeutsche mit vorherrschendem sprehhan neben vereinzeltem spehhan in meist oberdeutschen Glossen und das Mittelniederländische, wo man neben spreken als eine seltene Nebenform speken findet. Fasst man diese Schwankungen zwischen sprund $s p$ - im Anlaut des Verbs als Evidenz für eine Entwicklung von sprzu $s p$ - auf, kann man nicht umhin, diese Entwicklung als eine sporadisch auftretende Tendenz zu betrachten. Der Cluster spr- ist in allen Dialekten der betreffenden Sprachen sonst stabil. Die Fortsetzungen der anderen Lexeme mit altem spr-im Anlaut - wie zum Beispiel urgerm. *spreutan,sprießen', *sprengan- , aufspringen' und *sprēan- ,sprühen' - decken mit ihren Flexionsformen und Derivaten sämtliche lautlichen Konstellationen ab, die im Paradigma von urgerm. *sprekan- möglich sind, weisen aber niemals $r$-lose Varianten auf. ${ }^{20}$

ðā ärenan and scyttelas ðā īrenan gebrac gl. contriuit portas aereas et uectes ferreos confregit ,zerdrückte das eherne Tor und zerbrach die eisernen Sperrbalken' (PsGlB Brenner 106.16). Ahd. sluzzil übersetzt bei Tatian (90.3 und 141.11) und in Glossen (vgl. Wells 1990: 561) ausschließlich lat. clauis. Auch as. slutil glossiert lat. clauis (99.27 nach Wadstein 1899). Es scheint klar, dass ae. scyttel den Riegel bezeichnete, der zum Verschließen einer Tür von innen diente. Mit ahd. sluzzil und as. slutil wurde dagegen offenbar der eiserne Haken bezeichnet, der zum Zu- oder Aufsperren einer Tür von a u ßen verwendet wurde. Es ist prinzipiell nicht ausgeschlossen, dass sowohl ae. scyttel ,Riegel' als auch ahd. sluzzil, as. slutil ,Schlüssel` auf ein und dasselbe Wort zurückgehen. Diese Annahme ist aber keineswegs zwingend oder naheliegend. Es ist einfacher, ae. scyttel als eine einzelsprachliche Suffixableitung von ae. scyttan $<$ urgerm. *skutjanaufzufassen, was auch das geminierte $t t$ von scyttel erklärt. Ahd. sluzzil und as. slutil wären dagegen vom starken Verb urgerm. *sleutan- abgeleitet, das in ahd. sliozan fortgesetzt ist.

20. Gelegentlich wird vorgeschlagen, an urgerm. *spreutan- ,sprießen ${ }^{*}$ das germanische Wort für Jagdspeer *speuta- $\mathrm{m}$. oder $\mathrm{n}$. anzuschließen, das aufgrund von ahd. spioz m., as. -spiot und awn. spiōt n. rekonstruiert wird. Die semantische Distanz zwischen den Begriffen ,sprießen' und ,Jagdspeer' spricht gegen die etymologische Verwandtschaft der beiden Wörter. Semantisch ansprechend ist der Fall ae. sprac gl. sarmentum neben ahd. spah gl. sarmentum, cremium (also ,dürres, zerkleinertes Brennholz', ,Reisig $)$ ). Das althochdeutsche Wort, das auch schwach flektiert (ahd. spahho und spahha in Glossen), kann aber Substantivierung des Adjektivs sein, das in mnd. und mnl. spak ,dürr, 
Dieser letzte Umstand spricht aber gleichermaßen gegen jede Erklärung der Variation $s p r-\sim s p$ - beim westgermanischen Wort für ,sprechen ${ }^{6}$ durch eine lautliche Entwicklung. Die Spezialisierung eines Lautwandels, ob regelmäßig oder nicht, auf ein einziges Lexem ohne signifikante lautliche Besonderheiten wäre prinzipiell durch semantische Faktoren zu motivieren, was in jeder Theorie des Lautwandels nur bei klaren Onomatopoetika oder ganz besonders frequenten Wortformen möglich ist.

Aus diesem Grund wurde in der Forschung mehrmals vorgeschlagen, mit zwei ursprünglich verschiedenen Verben *sprekan- und *spekan- zu rechnen, die uns aufgrund ähnlicher Lautung und ähnlicher Semantik bei der Analyse der Texte als Varianten ein und desselben Verbs vorkommen, sich in den betreffenden Dialekten gegenseitig beeinflussen oder auch verdrängen konnten. Diese Hypothese setzt natürlich voraus, dass man für die beiden Verben für ,sprechen' eine Quelle angeben kann. Bei der Suche nach den Quellen muss man allerdings berücksichtigen, dass es sich bei *sprekan- und *spekan- kaum um genuine Verben für den Begriff ,sprechen' oder ,sagen' handeln kann. Für diesen Begriff war im Urgermanischen ursprünglich das starke Verb $* k^{w}$ epan- zuständig, das nicht nur in den westgermanischen Sprachen (ahd. quedan, ae. cweðan), sondern auch im ostgermanischen Gotisch (qiban) und den nordgermanischen Dialekten (awn. kveða) als das eigentliche Verb für ,sprechen' und ,sagen' fungiert und somit in dieser Funktion alt sein muss.

Die $r$-haltige Variante westgermanisch *sprekan- verbindet man traditionell mit der Wortfamilie des altwestnordischen schwachen Verbs spraka ,knistern, prasseln`. Die baltische Entsprechung litauisch spragù ,knistern, prasseln' weist auf eine alte Verbalwurzel hin, die für die vorgermanische Zeit als *spreg rekonstruiert werden kann. Die semantische Entwicklung von ,knistern, prasseln ${ }^{6} \mathrm{zu}$,sprechen ${ }^{6}$ findet zahlreiche Parallelen in verschiedenen Sprachen der Welt. Man vgl. zum Beispiel russisch treščát" ,schnell sprechen', dessen eigentliche Bedeutung ebenfalls ,knistern' ist. Ferner kann man auf deutsch ratschen ,schwatzen" hinweisen, das ursprünglich ,klappern' bedeutete.

Die $r$-lose Variante westgerm. *spekan- kann man unter der Annahme eines „beweglichen $s^{\prime \prime}$ im Anlaut direkt aus indogermanisch * $b^{h} e^{\prime} g^{\prime}-e$ - herleiten. Zur Entwicklung vorgerm. ${ }^{*} s b^{h_{-}}>$urgerm. ${ }^{*} s p$ - vgl. die bekannten Fälle des Typs vorgerm. * $d^{h}$ óumo- m. ,Dampf ${ }^{*}>$ urgerm. *daumam. (ahd. toum, mnl. doom) neben vorgerm. ${ }^{*} d^{h}$ óumo- m. ,Dampf ${ }^{\text {" }}>$

trocken' fortgesetzt ist und etymologisch wahrscheinlich zur Wurzel von ae. bacan, ahd. bahhan ,rösten, backen, dörren" gehört (mit ,,beweglichem $s^{“}$ ). Ae. sprac (mit anderer Ablautstufe auch mnd. sprok gl. sarmenta) ist dagegen mit der Wurzel von serbischkirchenslavisch prǔžiti, pražiti ,rösten, dörren' zusammenzustellen. In beiden Fällen muss man wieder mit ,beweglichem $s$ " rechnen. 
urgerm. * stauma- m. (ae. stēam, mnl. stoom). Die Semantik von * $b^{h} e^{\prime}{ }^{\prime}-$ $e$ - geht am klarsten aus der Verwendung seiner altindischen Fortsetzung bhája- hervor, die im Aktiv ,zuteilen' und im Medium ,als Anteil bekommen' bedeutet. Die Kategorie der Diathese ist im West- und Nordgermanischen vollständig aufgegeben, so dass die alten semantischen Unterschiede hier nicht mehr an morphologische Merkmale gebunden sind. Unter diesen Umständen kann man erwarten, dass ,zuteilen' und ,als Anteil bekommen' in einer neuen Semantik verschmelzen konnten, etwa ,verhandeln'. Eine mögliche Spur des primären Verbs *spekan-, verhandeln' außerhalb des Westgermanischen kann man sehen im sonst isolierten Adjektiv altwestnordisch spak-r, das auf einer Seite ,einsichtig, verständig', auf der anderen ,friedfertig, sanftmütig' heißt. Beide Bedeutungen des Adjektivs können aus einer gemeinsamen ursprünglichen Bedeutung etwa ,gesprächsbereit' stammen, dem Gegensatz einer kompromisslosen Aggression. Zur angenommenen semantischen Entwicklung von etwa ,verhandeln' $\mathrm{zu}$,sprechen' bei urgerm. *spekan- vgl. als typologische Parallele urgerm. *rēdan- ,beraten' (got. ga-rēdan, ae. rēedan, ahd. rātan, awn. räða), dessen Derivat got. rōdjan ,reden' oder ,sprechen' bedeutet.

Westgerm. *sprekan- ,knistern' $>$,sprechen' und *spekan-, verhandeln ${ }^{6}>$,sprechen ${ }^{6}$ werden in den westgermanischen Einzelsprachen wegen der Ähnlichkeit in Lautung und Semantik zu Varianten, die nicht mehr auseinander zu halten sind. In späterer Zeit setzt sich in den einen Sprachen die Fortsetzung des $r$-haltigen *sprekan-, in den anderen die des $r$-losen *spekan- durch. So erklärt sich der Befund, ohne dass die Annahme eines singulären Lautwandels ${ }^{*} s p r->{ }^{*} s p$ - im Westgermanischen notwendig wäre.

Zusammenfassend kann man festhalten, dass die Ablehnung des traditionellen Postulats der regelmäßigen Wirkung der Lautwandel in der Arbeit Vennemann (2000) weder aus den Grundannahmen der Präferenztheorie folgt noch durch empirisches Material im untersuchten Bereich der historischen Phonologie der germanischen Sprachen gefordert wird.

\section{Zusammenfassung}

Das Ziel der Präferenzrichtung in der historischen Phonologie besteht darin, die Motivation der konkreten Lautwandel in natürlichen Sprachen aufzudecken. Ob dieses Ziel erreicht werden kann, hängt davon $a b$, ob es gelingt, die universellen Präferenzen zuverlässig zu ermitteln. Beide Verfahren, die heute zur Ermittlung der Präferenzen angewendet werden - typologische Beobachtungen auf einer Seite, Untersuchung der Lautwandel, die durch diese Präferenzen hervorgerufen sein sollen, auf der anderen -, führen allerdings nicht $\mathrm{zu}$ annehmbaren Ergebnissen. 
Modifikationen, die von Anhängern der Präferenzrichtung am traditionellen Postulat der regelmäßigen Wirkung der Lautwandel vorgenommen werden, folgen nicht aus den Grundannahmen der Präferenztheorie. Die Berechtigung dieser Modifikationen ist auf empirischem Weg zu erweisen, was bisher nicht geschehen ist.

Eingereicht: 13. Januar 2007

Überarbeitete Fassung eingereicht:

16. Februar 2009
Lehrstuhl für Historische und Indogermanische Sprachwissenschaft Universität München

\section{Literatur}

Anderson, Stephen (1981). Why phonology isn’t „,natural“. Linguistic Inquiry 12: 493-539.

Auer, Peter (2001). Einige Argumente gegen die Silbe als universale prosodische Hauptkategorie. In Phonology. Critical Concepts. Vol. III. Syllables and Multi-level Analyses, Charles W. Kreidler (ed.), 121-142. London, New York: Routledge.

Blevins, Juliette (2004). Evolutionary Phonology: the Emergence of Sound Patterns. Cambridge: Cambridge University Press.

Blevins, Juliette (2005). Understanding antigemination. In Linguistic Diversity and Language Theories, Zygmunt Frajzyngier, Adam Hodges \& David S. Rood (eds.), 203-234. Amsterdam, Philadelphia: Benjamins.

Blevins, Juliette (2006). A theoretical synopsis of Evolutionary Phonology. Theoretical Linguistics 32: 117-165.

Blevins, Juliette (2008). Consonant epenthesis: natural and unnatural histories. In Linguistic Universals and Language Change, Jeff Good (ed.), 79-107. Oxford: Oxford University Press.

Blevins, Juliette \& Andrew Garrett (1998). The origins of consonant-vowel metathesis. Language 74: $508-556$.

Dolbey, Andrew E. \& Gunnar Ó. Hansson (1999). The source of naturalness in synchronic phonology. Chicago Linguistic Society 35: 59-69.

Donegan, Patricia (1993). On the phonetic basis of phonological change. In Historical Linguistics: Problems and Perspectives, Charles Jones (ed.), 98-130. London: Longman.

Donegan, Patricia \& David Stampe (1979). The study of natural phonology. In Current Approaches to Phonological Theory, Daniel A. Dinnsen (ed.), 126-173. Bloomington: Indiana University Press.

Dressler, Wolfgang U. (1987) (ed.). Leitmotifs in Natural Morphology. Amsterdam, Philadelphia: Benjamins.

Dziubalska-Kołaczyk, Katarzyna (2001). Phonotactic constraints are preferences. In Constraints and Preferences, Katarzyna Dziubalska-Kołaczyk (ed.), 69-100. Berlin, New York: Mouton de Gruyter.

Gess, Randall (2003). On re-ranking and explanatory adequacy in a constraint-based theory of phonological change. In Optimality Theory and Language Change, D. Eric Holt (ed.), 67-90. Dordrecht, Boston, London: Kluwer.

Gibbon, Dafydd (2001). Preferences as defaults in computational phonology. In Constraints and Preferences, Katarzyna Dziubalska-Kołaczyk (ed.), 143-200. Berlin, New York: Mouton de Gruyter.

Hale, Mark (2003). Neogrammarian sound change. In The Handbook of Historical Linguistics, Brian D. Joseph \& Richard D. Janda (eds.), 343-368. Oxford: Blackwell.

Hale, Mark (2007). Historical Linguistics: Theory and Method. Oxford: Blackwell.

Ham, William H. (1998). A new approach to an old problem: Gemination and constraint reranking in West Germanic. Journal of Comparative Germanic Linguistics 1: 225-262. 
Hayes, Bruce (1999). Phonetically driven phonology: the role of optimality theory and inductive grounding. In Functionalism and Formalism in Linguistics. Vol. I. General Papers, Michael Darnell, Edith A. Moravcsik, Michael Noonan, Frederick J. Newmeyer \& Kathleen M. Wheatley (eds.), 243-285. Amsterdam, Philadelphia: Benjamins.

Hill, Eugen (im Druck). Lautgesetze und Dialektvariation in den Jenissej-Sprachen. Entwicklung der Labiale im Ketischen und Jugischen. Ural-Altaische Jahrbücher.

Hoffmann, Karl \& Bernhard Forssman (2004). Avestische Laut- und Flexionslehre. Innsbruck: Institut für Sprachen und Literaturen der Universität Innsbruck, 2., durchgesehene und erweiterte Auflage.

Holt, D. Eric (2003) (ed.). Optimality Theory and Language Change. Dordrecht, Boston, London: Kluwer.

Kager, René (1999). Optimality Theory. Cambridge: Cambridge University Press.

Kiparsky, Paul (1988). Phonological change. In Linguistics: The Cambridge Survey. Vol. I: Linguistic Theory, Frederick J. Newmeyer (ed.), 363-415. Cambridge: Cambridge University Press.

Kirchner, Robert (2000). Geminate inalterability and lenition. Language 76: 509-545.

Lieberman, Stephen J. (1991). The regularity of sound change: a Semitistic perspective. In Patterns of Change, Change of Patterns: Linguistic Change and Reconstruction Methodology, Philip Baldi (ed.), 299-323. Berlin, New York: Mouton de Gruyter.

Loporcaro, Michele (2001). Rules vs. constraints in modeling phonological change: the case of Raddoppiamento Fonosintattico. In Constraints and Preferences, Katarzyna Dziubalska-Kołaczyk (ed.), 269-290. Berlin, New York: Mouton de Gruyter.

Loporcaro, Michele (2007). Facts, theory and dogmas in historical linguistics. Vowel quantity from Latin to Romance. In Historical Linguistics 2005. Selected Papers from the $17^{\text {th }}$ International Conference on Historical Linguistics, Madison, Wisconsin, 31 July5 August 2005, Joseph Salmons \& Shannon Dubenion-Smith (eds.), 311-336. Amsterdam, Philadelphia: Benjamins.

Lutz, Angelika (1988). On the historical phonotactics of English. In Luick Revisited: Papers Read at the Luick Symposium at Schloß Liechtenstein 15.-18. 9. 1985, Dieter Kastovsky \& Gero Bauer (eds.), 221-239. Tübingen: Narr.

Lutz, Angelika (1991). Phonotaktisch gesteuerte Konsonantenveränderungen in der Geschichte des Englischen. Tübingen: Niemeyer.

Lutz, Angelika (1992). Lexical and morphological consequences of phonotactic change in the history of English. In History of Englishes. New Methods and Interpretations in Historical Linguistics, Matti Rissannen, Ossi Ihalainen, Terttu Nevalainen \& Irma Taavitsainen (eds.), 156-166. Berlin-New York: Mouton de Gruyter.

Macken, Marlys A. \& Joseph C. Salmons (1997). Prosodic templates in sound change. Diachronica 14: 31-66.

Mayerthaler, Willi (1981). Morphologische Natürlichkeit. Wiesbaden: Athenaion.

McMahon, April (2003.) On not explaining language change: Optimality Theory and the Great Vowel Shift. In Motives for Language Change, Raymond Hickey (ed.), 82-96. Cambridge: Cambridge University Press.

Murray, Robert W. (1982). Consonant cluster developments in Pāli. Folia Linguistica Historica 3: $163-184$.

Murray, Robert W. (1986). Urgermanische Silbenstruktur und die westgermanische Konsonantengemination. Beiträge zur Geschichte der deutschen Sprache und Literatur 108: $333-356$.

Murray, Robert W. (1988). Phonological Strength and Early Germanic Syllable Structure. München: Fink.

Murray, Robert W. (1991). Early Germanic syllable structure revisited. Diachronica 8: 201-238.

Murray, Robert W. (2000). Old problems, new approaches, and optimizing preferences: A reply to Ham (1998). Journal of Comparative Germanic Linguistics 3: 221-237. 
Murray, Robert W. \& Theo Vennemann (1982). Syllable contact change in Germanic, Greek and Sidamo. Klagenfurter Beiträge zur Sprachwissenschaft 8: 321-349.

Murray, Robert W. \& Theo Vennemann (1983). Sound change and syllable structure in Germanic phonology. Language 59: 514-528.

Prince, Alan \& Paul Smolensky (2004). Optimality Theory. Constraint Interaction in Generative Grammar. Oxford: Blackwell.

Ritt, Nikolaus (2001). Are optimality theoretical „constraints“ the same as natural linguistic „preferences“? In Constraints and Preferences, Katarzyna Dziubalska-Kołaczyk (ed.), 291-310. Berlin, New York: Mouton de Gruyter.

Schuchardt, Hugo (1885). Über die Lautgesetze. Gegen die Junggrammatiker. Berlin: Oppenheim.

Seebold, Elmar (1970). Vergleichendes und etymologisches Wörterbuch der germanischen starken Verben. The Hague, Paris: Mouton de Gruyter.

Smith, Laura Catharine (2007). The resalience of prosodic templates in the history of West Germanic. In Historical Linguistics 2005. Selected Papers from the $17^{\text {th }}$ International Conference on Historical Linguistics, Madison, Wisconsin, 31 July-5 August 2005, Joseph Salmons \& Shannon Dubenion-Smith (eds.), 351-365. Amsterdam, Philadelphia: John Benjamins.

Vennemann, Theo (1972). Hugo Schuchardt's theory of phonological change. In Schuchardt, the Neogrammarians, and the Transformational Theory of Phonological Change, Theo Vennemann \& Terence H. Wilbur (eds.), 115-179. Frankfurt: Athenäum.

Vennemann, Theo (1982). Zur Silbenstruktur der deutschen Standardsprache. In Silben, Segmente, Akzente. Referate zur Wort-, Satz- und Versphonologie anläßlich der vierten Jahrestagung der Deuschen Gesellschaft für Sprachwissenschaft, Köln, 2.-4. März 1982, Theo Vennemann (Hg.), 261-305. Tübingen: Niemeyer.

Vennemann, Theo (1983a). Überlegungen zu einer Theorie der linguistischen Präferenzen. Klangenfurter Beiträge zur Sprachwissenschaft 9: 262-92.

Vennemann, Theo (1983b). Causality in language change. Theories of linguistic preferences as a basis for linguistic explanations. Folia Liguistica Historica 4: 5-26.

Vennemann, Theo (1986a). Neuere Entwicklungen in der Phonologie. Berlin: Mouton de Gruyter.

Vennemann, Theo (1986b). Syllable based sound changes in Early Armenian. Annual of Armenian Linguistics 7: 27-43.

Vennemann, Theo (1988). Preference Laws for Syllable Structure and the Explanation of Sound Change. With special reference to German, Germanic, Italian, and Latin. Berlin: Mouton de Gruyter.

Vennemann, Theo (1993). Language change as language improvement. In Historical Linguistics: Problems and Perspectives, Charles Jones (ed.), 319-344. London: Longman.

Vennemann, Theo (2000). Triple-cluster reduction in Germanic: Etymology without sound laws? Historische Sprachforschung 113: 239-58.

Vine, Brent (1985). On the Germanic type lükan. Zeitschrift für Vergleichende Sprachforschung 98: 59-81.

Wadstein, Elis. (1899). Kleinere altsächsische Sprachdenkmäler mit Anmerkungen und Glossar. Norden, Leipzig: Soltau.

Wang, William S.-Y. (1969). Competing changes as a cause of residue. Language 45: 9-25.

Wang, William S.-Y. \& Chinfa Lien (1993). Bidirectional diffusion in sound change. In Historical Linguistics: Problems and Perspectives, Charles Jones (ed.), 344-400. London: Longman.

Wells, John C. (1990). Althochdeutsches Glossenwörterbuch einschließlich des von Prof. Dr. Taylor Starck begonnenen Glossenindexes. Heidelberg: Winter.

Wilbur, Terence H. (1972). Hugo Schuchardt and the Neogrammarians. In Schuchardt, the Neogrammarians, and the Transformational Theory of Phonological Change, Theo Vennemann \& Terence H. Wilbur (eds.), 75-113. Frankfurt: Athenäum. 
Wurzel, Wolfgang U. (1984). Flexionsmorphologie und Natürlichkeit. Ein Beitrag zur morphologischen Theoriebildung. Berlin: Akademie-Verlag.

Wurzel, Wolfgang U. (1985). Morphologische Natürlichkeit und morphologischer Wandel. Zur Vorhersagbarkeit von Sprachveränderungen. In Papers from the $6^{\text {th }}$ International Conference on Historical Linguistics, Jacek Fisiak (ed.), 587-599. Amsterdam: Benjamins \& Adam Mickiewicz University Press.

Wurzel, Wolfgang U. (1999). Principles of evaluation, change and related issues. Zeitschrift für Sprachwissenschaft 18: 242-250.

Yu, Alan C. L. (2004). Explaining final obstruent voicing in Lezgian: phonetics and history. Language 80: 73-97. 